\title{
Probabilistic model based on circular statistics for quantifying coverage depth dynamics originating from DNA replication
}

\author{
Shinya Suzuki ${ }^{1}$, Takuji Yamada ${ }^{\text {Corresp. } 1}$ \\ ${ }^{1}$ Department of Life Science and Technology, Tokyo Institute of Technology, Meguro, Tokyo, Japan \\ Corresponding Author: Takuji Yamada \\ Email address: takuji@bio.titech.ac.jp
}

Background. With the development of DNA sequencing technology, static omics profiling in microbial communities, such as taxonomic and functional gene composition determination, has become possible. Additionally, the recently proposed in situ growth rate estimation method allows the applicable range of current comparative metagenomics to be extended to dynamic profiling. However, with this method, the applicable target range is presently limited. Furthermore, the characteristics of coverage depth during replication have not been sufficiently investigated.

Results. We developed a probabilistic model that mimics coverage depth dynamics. This statistical model explains the bias that occurs in the coverage depth due to DNA replication and errors that arise from coverage depth observation. Although our method requires a complete genome sequence, it involves a stable to low coverage depth $(>0.01 \times)$. We also evaluated the estimation using real wholegenome sequence datasets and reproduced the growth dynamics observed in previous studies. By utilizing a circular distribution in the model, our method facilitates the quantification of unmeasured coverage depth features, including peakedness, skewness, and degree of density, around the replication origin. When we applied the model to time-series culture samples, the skewness parameter, which indicates the asymmetry, was stable over time; however, the peakedness and degree of density parameters, which indicate the concentration level at the replication origin, changed dynamically. Furthermore, we demonstrated the activity measurement of multiple replication origins in a single chromosome.

Conclusions. We devised a novel framework for quantifying coverage depth dynamics. Our study is expected to serve as a basis for replication activity estimation from a broader perspective using the statistical model. 


\section{Probabilistic model based on circular statistics for}

2 quantifying coverage depth dynamics originating from 3 DNA replication

6 Shinya Suzuki, Takuji Yamada

7

Department of Life Science and Technology, Tokyo Institute of Technology, 2-12-1 Ookayama, Meguro, Tokyo 152-8550, Japan

Corresponding Author:

Takuji Yamada

13 2-12-1 Ookayama, Meguro, Tokyo 152-8550, Japan 


\section{Abstract}

40 Background. With the development of DNA sequencing technology, static omics profiling in microbial communities, such as taxonomic and functional gene composition determination, has become possible. Additionally, the recently proposed in situ growth rate estimation method allows the applicable range of current comparative metagenomics to be extended to dynamic profiling. However, with this method, the applicable target range is presently limited. Furthermore, the characteristics of coverage depth during replication have not been sufficiently investigated.

Results. We developed a probabilistic model that mimics coverage depth dynamics. This statistical model explains the bias that occurs in the coverage depth due to DNA replication and errors that arise from coverage depth observation. Although our method requires a complete genome sequence, it involves a stable to low coverage depth $(>0.01 \times)$. We also evaluated the estimation using real whole-genome sequence datasets and reproduced the growth dynamics observed in previous studies. By utilizing a circular distribution in the model, our method facilitates the quantification of unmeasured coverage depth features, including peakedness, skewness, and degree of density, around the replication origin. When we applied the model to time-series culture samples, the skewness parameter, which indicates the asymmetry, was stable over time; however, the peakedness and degree of density parameters, which indicate the concentration level at the replication origin, changed dynamically. Furthermore, we demonstrated the activity measurement of multiple replication origins in a single chromosome.

Conclusions. We devised a novel framework for quantifying coverage depth dynamics. Our study is expected to serve as a basis for replication activity estimation from a broader perspective using the statistical model.

\section{Introduction}

The development of high-throughput DNA sequencers has enabled massive and exhaustive microbiome analyses. By mapping fragmented reads onto databases, the taxonomic and functional gene composition of a sample can be measured. Several researchers have utilized this procedure to investigate samples from various environments, such as those of human and animal bodies as well as those of other types of environmental samples (Hildebrand et al., 2013; Kato et al., 2015; Zhu et al., 2015; Higashi et al., 2018). One possible means to progressing beyond the profiling of static information involves investigating microbial dynamics. Although time-series microbiome profiling via quantitative polymerase chain reaction (PCR) or cell-sorting may allow dynamics measurement, such methods do not easily provide a comprehensive understanding of growth dynamics from the single sample involved therein (Tourlousse et al., 2017; Vandeputte et al., 2017). Meanwhile, the peak-to-trough ratio (PTR) of the coverage of whole genome sequencing (WGS) reads mapped to a reference genome sequence provides an estimate of 
79 This method is based on the considerable increase in DNA around the replication origin via 80 bidirectional DNA replication (Cooper \& Helmstetter, 1968; Bremer \& Churchward, 1977). As 81 quantitative pipelines continuously undergo re-evaluation and extension, a draft quality genome 82 sequence may be applied. Few methods have been proposed to quantify the growth of bacteria 83 from genomic data. iRep uses a mechanism in which the slope of the sorted coverage on contig 84 sequences was correlated with the growth rate (Brown et al., 2016). GRiD enables more robust estimation by sorting the coverage depths of multiple contigs (Emiola \& Oh, 2018). DEMIC performed accurate estimation by using the coverage depths of multiple samples and estimating the appropriate position via principal component analysis (Gao \& $\mathrm{Li}, 2018)$. Some studies using such pipelines have revealed associations between growth estimates and factors such as disease, 24-hour oscillations, and diet (Olm et al., 2017; Forsyth et al., 2018). Thus, such an approach quantifying the growth of bacteria from coverage depth is expected to facilitate the investigation of new fields of microbial research. However, some questions associated with coverage depth modeling remain unresolved.

The first challenge regarding growth estimation from coverage depth is related to the application scope of the method. Previous studies have enabled growth estimation for a broad range of samples, but the range of applicability remains limited. Taking coverage depth as an example, even the most robust method currently requires $0.05 \times$ average coverage with a complete sequence or $0.2 \times$ with a de novo-assembled sequence. A novel growth rate estimation method that is less sensitive to decreases in coverage depth could be utilized in broader applications. Second, the previously proposed pipelines are not applicable to microbes with multiple replication origins as these pipelines use a model based on a single peak and trough. This approach narrows the range of measurement targets as some taxa such as archaea have two or more replication origins in a single replicon (Lundgren et al., 2004; Robinson et al., 2004; Andersson et al., 2010). It has also been suggested that some bacteria have multiple replication origins (Gao, 2015; Ohbayashi et al., 2016). In addition to growth estimation based on coverage depth, it is also difficult to predict replication origins from sequence features such as GC-skew in some microbes (Gao \& Zhang, 2008; Sernova \& Gelfand, 2008; Vieira-Silva \& Rocha, 2010). To overcome this challenge, a previous report proposed a method for predicting the positions of multiple replication origins based on the amount of chromosomal DNA (Xu et al., 2012). However, no method with a statistical background has been introduced. Third, the characteristics of coverage depth distributions themselves have not been sufficiently investigated. Some previous studies have reported non-linear DNA quantity trends (Hawkins et al., 2013; Pelve et al., 2013; Wu et al., 2014; Akiyama et al., 2016), such as those including a significant increase around the replication origin. Based on these studies, it has been suggested that replication affects not only the ratio of maximum to minimum depth, but also changes the degree of density around the replication origin. Modeling this phenomenon could be useful for both molecular biologists and microbiologists, enabling them to quantify the extra dynamics of replication. Furthermore, it is unclear whether the coverage depth trend is skewed toward the $5^{\prime}$ direction, is skewed toward the 3 ' direction, or is symmetric. As some previous studies have suggested that 
119 the asymmetry of replisome progression is associated with the phenotype (Rodriguez-Lopez et

120

121

122

123

124

125

126

127

128

129

130

131

132

133

134

135

136

137

138

139

140

141

142

143

144

145

146

147

148

149

150

151

152

153

154

155

156

157

al., 2002), it would be valuable to develop a method of symmetric level detection.

Here, we propose a method of modeling coverage depth dynamics using probabilistic statistics. Focusing on data generation when mapping fragmented reads to a circular genome sequence, we combined multinomial and directional distributions to mimic the read sampling process and bias of the DNA quantity. When applied to a dataset from a culture experiment, our method provided a stable and robust estimation of even a small number of reads and mutated reference sequences. To observe the degree of correlation between the growth estimates and experimental growth rates, we applied our method to WGS reads, which were obtained from a previous time-series culture experiment (Korem et al., 2015); this led to the observation of a high degree of correlation between the growth estimates and experimental growth rates. In vivo data sets were used to confirm the reproducibility of the growth dynamics in previous studies. Using the previous in vitro and in vivo samples, we ensured that our method is sufficiently robust to coverage and noise. Furthermore, by extending these models to enable them to form tapered and skewed coverage depth shapes, we designed a method of measuring coverage depth bias. Using a mixture of directional distributions allows growth estimation to be applied to sequences with multiple replication origins. We also demonstrate such estimations in relation to genome sequences of Sulfolobus solfataricus and Haloferax volcanii (McCarthy et al., 2015).

\section{Materials \& Methods}

\section{Circular distributions and statistics}

The distributions and statistics used in this study are shown in Table 1. The location parameter has the highest probability and corresponds to the replication origin in the chromosome in this model. We changed the character of the concentration parameter by changing the distribution, as it can be aligned by $\rho_{\mathrm{c} .}=\frac{\tanh (\kappa)}{2}$ or $\rho_{\text {w.C. }}=\tanh \left(\frac{\kappa}{2}\right)$ (Jones \& Pewsey, 2005; Pewsey, Neuhäuser \&

Ruxton, 2013). In addition to major distributions, we introduced a linear cardioid distribution and exponential linear cardioid distribution to evaluate the coverage depth trend. These functions are symmetric around the location parameter, and the integral around a unit circle is 1; i.e., $\int_{-\pi}^{\pi} P(\theta \mid \mu, \rho) d \theta=\int_{0}^{2 \pi} P(\theta \mid \mu, \rho) d \theta=1$. For each distribution, the probabilistic PTR can be analytically defined as the ratio between the maximum and minimum value of the probability density function (see the Statistical model to estimate replication rate section for details). Some of the models (von Mises, cardioid, wrapped Cauchy, and Jones-Pewsey) were symmetrically or asymmetrically extended with or without inverse transformation, as previously described (Abe, Pewsey \& Shimizu, 2013; Pewsey, Neuhäuser \& Ruxton, 2013; Abe, Pewsey \& Fujisawa, 2013). To make the shape near the mode of a distribution variable, we used Batschelet or inverse-Batschelet transformation. Batschelet transformation (symmetric extension; SE) transforms the angular variable into $g_{\lambda}(\theta)=(\theta-\mu)+\lambda \sin (\theta-\mu)$, where $\lambda$ is the peakedness parameter. Using this transformation, the normalization constant was calculated using the composite Simpson's law as the integral around a unit circle cannot be maintained as 1 . Inverse- 
158 Batschelet transformation (inverse symmetric extension; InvSE) transforms the angular variable

159

160

161

162

163

164

165

166

167

168

169

170

171

172

173

174

175

176

177

178

179

180

181

182

183

184

185

186

187

188

189

190

191

192

193

194

195

196 into $g_{\lambda}(\theta)=\frac{1-\lambda}{1+\lambda} \theta+\frac{2 \lambda}{1+\lambda} t_{1, \lambda}^{-1}(\theta)$, where $t_{1, \lambda}(\theta)=\theta-\frac{1}{2}(1+\lambda) \sin (\theta-\mu)$. This transformation does not change the normalization constant. To make the distribution asymmetric with modeinvariance, we used the mode-invariance asymmetric transformation extension (MIAE) or inverse-transformed mode-invariance asymmetric transformation extension (InvMIAE). These transformations satisfy the requirement that asymmetricity be analyzed in replication. As replication begins at the origin irrespective of the rapidity of bacterial growth, the highest coverage depth position is preserved regardless of the asymmetry level. The skewness parameter must not affect the pPTR when the pPTR and skewness are measured independently. In these transformations, the symmetricity around the mode changes via an additional skewness parameter, while the location parameter, concentration parameter, and pPTR remain unchanged even if the skewness parameter changes. MIAE transforms the angular variable into $g_{v}(\theta)=\theta-v$ $\sin ^{2}(\theta-\mu)$, where $v$ is the skewness parameter. This transformation also requires a normalization constant. InvMIAE transforms the angular variable into $g_{v}(\theta)=s_{v}^{-1}(\theta)$, where $s_{v}(\theta)=\theta+v \sin ^{2}$ $(\theta-\mu)$. This transformation does not change the normalization constant, and the position of the mode is preserved. To compute the inverse transformation, we used several root-finding algorithms (see Parameter estimation section). The significance of fitness improvement via additional parameters was evaluated using the likelihood ratio test with a chi-squared distribution based on the theorem of Wilks in addition to the Akaike Information Criterion (AIC) and the Bayes Information Criterion (BIC). To compute the likelihood ratios, the original distribution was compared with the extended distributions. The Jones-Pewsey distribution was compared with the von Mises distribution.

\section{Statistical model to estimate replication rate}

A statistical model that simulates the coverage depth dynamics along the genome position was constructed. Let $d_{i, s}$ be the coverage depth of the $i$ th position obtained when mapping the WGS reads of sample $s$ onto the genome sequence. Here $i$ represents the binned position of coverage. If the coverage depth is not compressed or binned, $i$ matches a nucleotide position. We fit a generalized linear model (GLM) for each $d_{i, s}$ as follows.

The starting point of our model is the conversion of $d_{i, s}$ into the frequency of observation of the $i$ th position. Here, the total number of observations $T_{s}$ for sample $s$ is calculated as the sum of $d_{i, s}$ over sequence length, $I$, i.e., $T_{s}=\sum_{i=1}^{I} d_{i, s}$. We did not directly fit the coverage depth to a model because it could fail to fit with a low coverage depth dataset. Instead, we modeled a bias to observe the base positions by mapping reads with a probability distribution $P$ and parameter set $\omega$, which defines the potential of observation probability, $i_{t, s} \sim P\left(\omega_{s}\right)$, where $t$ is a unique identifier of nucleotides for all reads mapped to the genome. Focusing on the genome structure of bacteria and archaea, the observation probability is supposed to be circular. For compatibility with the structure, the position $i$ is converted into an angle $\theta$, following $\theta=\frac{i}{I} 2 \pi$. Here, the coverage depths 
$197 d_{i}$ are stacking counts of the observed angle $\theta$. Circular statistics, instead of an ordinal real-value 198 approach, are required to quantify the bias based $\theta$. In circular statistics, the first possible means

199

200

201

202

203

204

205

206

207

208

209

210

211

212

213

214

215

216

217

218

219

220

221

222

223

224

225

226

227

228

229

230

231

232

233

234 of analyzing an angle dataset involves expressing the bias as a simple statistic without any model. For example, the mean resultant length (MRL) represents how data are concentrated around the sample mean direction. The second approach, which was mainly used in this study, involves the modeling of a phenomenon via probability distributions that generate positions. We introduced the following four distribution types from the circular distribution family: von Mises, wrapped Cauchy, cardioid, and Jones-Pewsey distributions. These distributions are widely used in circular statistics and are versatile in terms of implementation and inference (Jones \& Pewsey, 2005; Pewsey, Neuhäuser \& Ruxton, 2013). Additionally, these distributions are useful for representing known coverage depth characteristics. For example, some researchers have described non-linear coverage depth trends over genome sequences in both bacteria and archaea (Chen et al., 2005; Watanabe et al., 2012; Hawkins et al., 2013; Pelve et al., 2013; Rudolph et al., 2013; Wendel, Courcelle \& Courcelle, 2014; Wu et al., 2014; Maduike et al., 2014; Yang et al., 2015; Akiyama et al., 2016; Ohbayashi et al., 2016; Forsyth et al., 2018; Retkute et al., 2018). It was expected that this trend could be quantified by extension for the distributions proposed in the previous studies. For these circular distributions with likelihood $L_{\text {dist. }}$, the overall log-likelihood $\log L$ of the model can be calculated as follows:

$$
\log L(\omega \mid \theta, d, I, S)=\sum_{s=1}^{S} \sum_{i=1}^{I} d_{i, s} \log L_{\text {dist. }}\left(\omega \mid \theta_{i}\right)
$$

As $\theta$ is considered to be continuous rather than discrete for the purpose of these distributions, we confirmed that the parameters could be estimated appropriately (Supplementary Text S1). Equation (1) coincides with a term that changes with the probability parameter in the loglikelihood equation of the multinomial distribution shown in equation (2) although the sum of the probability over all nucleotides is not 1 because the distribution is not discrete:

$$
\log \text { Multinomial }(d \mid T, p)=\sum_{s=1}^{S} \sum_{t=1}^{T}{ }_{s} \log t-\sum_{s=1}^{S} \sum_{i=1}^{I} \sum_{j=1}^{d_{s, i}} \log j+\sum_{s=1}^{S} \sum_{i=1}^{I} d_{s, i} \log p_{i}
$$

Following the model with the likelihood represented by equation (1), the location parameter corresponds to the position of the replication origin as long as the concerned chromosome does not have multiple replication origins. Contrastingly, the concentration parameter is associated with growth as it determines the shape of the distribution. Therefore, we allowed the location parameter to be shared among all of the samples and the concentration parameter for each sample to be independent. For the concentration parameters, we set the halfStudent's $t$-distribution as a prior distribution (Gelman, 2006). We set 2.5 as the shape parameter; 0 as the location parameter; and 0.2 (von Mises, Jones-Pewsey), 0.1 (cardioid), 0.17 (wrapped Cauchy), 0.105 (linear cardioid), and 0.1103(exponential linear cardioid) as the scale parameters. These were selected such that the value of the cumulative probability density function became nearly 0.8 when the PTR was 2.0. This characteristic suggests that most of the PTRs are distributed between 1.0 and 2.0 in an environment. The distribution of the coverage depth PTR in a previous study rationalizes this suggestion (Korem et al., 2015). For the degree of 
235 density of the Jones-Pewsey distribution, the peakedness of the symmetric extended distribution,

236 and the skewness parameter of the asymmetry extended distribution, we set the Gaussian

237 distribution with a location parameter of 0 and a scale parameter of 1.0 as the prior distribution to

238 avoid overfitting. From the model, we introduced an estimate that expresses the degree of

239 growth. It is known that many microbes in prokaryotes replicate their chromosomal DNA on

240 both sides from the origin such that the apparent amount of DNA increases near the origin. This

241 behavior introduces a latent bias that makes DNA near the origin more likely to be observed

242 during replication. This bias is simply expressed by the concentration parameter in a circular

243 distribution. However, for consistency with the previous study (Korem et al., 2015), we defined a

244 probabilistic PTR (pPTR), which is the ratio of the maximum of probability density function to

the minimum, i.e., $P T R_{\text {probability }}=\frac{p_{\max }(\theta)}{p_{\min }(\theta)}$, as a growth dynamics index. This score represents the

latent bias of the probability for the position at which a nucleotide is observed around the replication origin. Unlike the original PTR, which is directly estimated from the coverage depth, pPTR is obtained by modeling the bias based on a circular distribution and the probability framework of interest. Following the model, in which the coverage depth is a result of discrete sampling expressed as in equation (2), the coverage depth at a given position can be modeled by the binomial distribution by formula (3):

$$
d_{i, s} \sim \operatorname{Binomial}\left(T_{s}, P\left(\theta=\frac{i}{I} 2 \pi \mid \omega_{s}\right)\right) .
$$

253

254

255

256

257

258

259

260

261

262

263

264

265

266

267

268

269

270

This equation hints at the benefit of using probability rather than coverage depth directly (Supplementary Text S2; Supplementary Fig. 1).

\section{Extending the model to multiple origins of replication}

We constructed a statistical model for multiple origins of the replication-based mixture model and circular distributions. Let $\alpha_{m}$ be the ratio of the mixture to the $m$ th replication origin and $M$ be the number of the replication origin, then the probability of obtaining the angle $\theta$ from the sample $s$ is formulated to $P\left(\theta \mid \omega_{s}, \alpha\right)=\sum_{m=1}^{M} \alpha_{m} P_{\text {circle }}\left(\theta \mid \omega_{s, m}\right)$ with the circular distribution $P_{\text {circle. }}$. Note that the sum of the ratio is 1, i.e., $\sum_{m=1}^{M} \alpha_{m}=1$. Based on the model, the overall loglikelihood $\log L$ can be calculated as follows:

$$
\log L(\omega, a \mid \theta, d, I, S) \propto \sum_{s=1}^{S} \sum_{i=1}^{I} d_{i, S} \log \left(\sum_{m=1}^{M} \alpha_{m} L_{\text {dist. }}\left(\omega_{m, S} \mid \theta={ }_{i}^{i} 2 \pi, s=s\right)\right) .
$$

The equation is compatible with a genome sequence with a single replication origin as it takes the same form as equation (1) when we set $\alpha_{1}=1$ and $M=1$. We set a Dirichlet distribution as the prior distribution of $\alpha$ as $\alpha \sim \operatorname{Dirichlet}(A)$ and employed $50 / M$ as $A$, as previous studies have implied that each replication origin shows similar activity (Robinson et al., 2004; Andersson et al., 2010; Hawkins et al., 2013). Then, the ratio is likely to assume a similar value, which defines the equality of the mixture. As the activity index for multiple replication origins, we defined a 
271 weighted pPTR (wPTR) and mean-weighted pPTR (mwPTR). The wPTR of the $m$ th replication

272 origin is computed via a weighted concentration parameter using a mixture ratio, where mwPTR

273 is the average of these. For example, the wPTR of the von Mises-based model is given by $\exp (2$

$274 \alpha_{m} \kappa_{m}$ ), and mwPTR is given by $\frac{1}{M} \sum_{m=1}^{M} w P T R_{m}$. These scores are based on the model that

275 replication stops if the replisome comes across another replisome, as mainly reported in

276 prokaryotes (Leman \& Noguchi, 2013; Wendel, Courcelle \& Courcelle, 2014); however, this

277 model has not been sufficiently investigated in archaea or eukaryotes. This model assumes that

278 the effect of multiple origins at each location can be expressed as their sum. Following the mixed

279 effects of multiple origins, the coverage depth is probabilistically sampled. This assumption

280 results in the probability of regions that are not related to the origin approaching low values.

281 Hence, the probability distribution of each origin becomes very steep, with increases in the

282 concentration parameter and unweighted PTR. By weighting the parameters, we approximated

283 the degree of activity in the case in which only the single origin worked in the chromosome. We

284 used the average of the WPTR as the representative score of the chromosome because a previous

285 study reported that the growth rate decreased when a replication origin on the chromosome was

286 knocked out, whereas the deviation of the DNA amount between the origins and terminus did not

287 change significantly (Wu et al., 2014). By using the average, the effect of activation on multiple

288 origins could be determined.

289

290

291

292

293

294

295

296

297

298

299

300

301

302

303

304

305

306

307

308

309

\section{Parameter estimation}

For all data, we estimated the model parameters using an implemented software package. This package fits the parameters to the data by maximizing the joint posterior (optimizing mode) or generating samples from the posterior distribution of the parameters (sampling mode). Briefly, after maximizing the log-likelihood of the model for the data via each method, we adopted the value that yielded the maximum log-likelihood via the optimizing mode or the expected $a$ posteriori (EAP) of the parameter posterior distribution via the sampling mode. Unless otherwise noted, we used the limited-memory Broyden-Fletcher-Goldfarb-Shanno (L-BFGS) algorithm for the optimizing mode and the No-U-Turn Sampler (NUTS) algorithm (Hoffman \& Gelman, 2014), which is the quasi-Markov chain Monte Carlo (MCMC) method, for the sampling mode. For sampling, we set the number of chains to 1 and the number of iterations to 500; the first 300 iterations were considered a warm-up and discarded. From the samples, we calculated the EAP as a representative model parameter. The convergence of the sampling was checked using Rhat statistics. If the statistics were equal to or less than 1.1, the result was accepted (Gelman et al., 2013). We used composite Simpson's algorithm with 20 subintervals to calculate the integrals in the models. We computed the inverse transformation of the functions using Newton's method when the skewness $v$ or peakedness parameter $\lambda$ was less than or greater than 0.8 . These thresholds were evaluated by performing manual simulations such that the transformations did not oscillate. We checked the convergence of the function as to whether the error was less than the machine precision of a float-type variable defined using Stan. The Illinois method was used 
311 in other cases (Dowell \& Jarratt, 1971). Then, we checked the convergence of the function, as to

312 whether the error was less than $1.0 \times 10^{-13}$. In both methods, we defined a maximum iteration

313 count that terminated the calculation at 30 iterations for Newton's method and 100 iterations for

314 the Illinois method. We set the mathematics transformation to reasonably estimate the

315 parameters. If a location parameter is estimated directly from 0 to $2 \pi$, and the true location

316 parameter is located near the edge of the range, the parameter estimation is likely to fail as it

317 does not detect cyclicity. We re-parameterized the location parameter as $\mu=\arctan 2\left(\overrightarrow{\theta_{\mu}}\right)$ for the

318 two-dimensional unit vector $\overrightarrow{\theta_{\mu}}$ to overcome this estimation difficulty (Pewsey, Neuhäuser \&

319 Ruxton, 2013). The unit vector can be estimated directly as each element has continuity from -1

320 to 1 . To calculate information criteria, $1+$ number of the sample $\times 2$ was used as the number of

321 parameters for the Jones-Pewsey distribution-based model, and 1+number of the samples was

322 used for the others.

323

324

325

326

327

328

329

330

331

332

333

334

335

336

337

338

339

340

341

342

343

344

345

\section{Coverage depth calculation}

The coverage depth was calculated by aligning the WGS reads to the template genome sequence. We downloaded WGS via the SRA Toolkit. After converting the WGS reads into the FASTQ format, we aligned them to the genome sequence using Bowtie2 with a "--very-sensitive" parameter set (Langmead \& Salzberg, 2012). We sorted the resulting SAM files and calculated the depth using SAMtools (Li et al., 2009). Next, a moving median filter with a $100 \mathrm{nt}$ window size and a $100 \mathrm{nt}$ stride length was applied. The moving median filter runs through the coverage depths, replacing each coverage with the median of neighboring locations. We applied the moving median filter for the following two reasons: to reduce the noise and outliers and to reduce the data size, which affects the computational time of the model fitting. If the coverage depth seemed to have noise regions that increased the coverage due to highly conserved regions such as ribosomal genes, an additional filtering for outliers was performed; specifically, the top $1 \%$ of the coverage depth was removed and replaced with blank coverage. This decision and the threshold, which were determined based on a previous study (Brown et al., 2016), were independently evaluated using a frequency histogram of the coverage depth containing a noise region, which increases the coverage in multiple datasets (Supplementary Text S3). Certain regions that remained blank following filtering were filled with 0 . As the WGS reads of $H$. volcanii were separated into multiple FASTQ files, we concatenated them into a single file based on growth conditions prior to alignment. To evaluate the error in the sequence edge parts, we copied $263 \mathrm{nt}$ in the head portion of the genome sequence to the tail portion. We also constructed a graph genome sequence, circularized it, and mapped WGS reads to the graph genome sequence using the variation graph toolkit (vg) with the default parameter set (Garrison et al., 2018). We omitted the GC-content correction procedure to simplify the pipeline as the method using the full-length genome sequence seemed to be robust (Brown et al., 2016) against the local coverage depth bias attributed to the GC-content (Ross et al., 2013). If a user requires greater accuracy, we 
350 recommend using the correction method or PCR-free library preparation (Benjamini \& Speed, 351 2012; Brown et al., 2016; Gao \& Li, 2018).

352

353

354

355

356

357

358

359

360

361

362

363

364

365

366

367

368

369

370

371

372

373

374

375

376

377

378

379

380

381

382

383

384

385

386

387

\section{Growth rate evaluation with experimental growth rate}

The accuracy of the growth estimates was evaluated via comparisons with experimental growth rates. Unless otherwise noted, the experimental growth rates were calculated from the colony formation unit $(\mathrm{CFU}) / \mathrm{ml}$, optical density (OD), or relative abundance using $g r_{i}=$ $\frac{\log _{2}\left(a b u n_{i+1}\right)-\log _{2}\left(a b u n_{i-1}\right)}{t_{i+1}-t_{i-1}}$ following the approach taken in a previous study (Korem et al., 2015); e.g., the experimental growth for Supplementary Fig. 3c uses this equation. For relative abundance, we used the mOTUs2 pipeline with the default parameter set (Milanese et al., 2019). When comparing methods, we additionally calculated the experimental growth rate (shown in Supplementary Fig. 3d) in a differential manner to obtain the dynamics in a short time span; i.e., $g r_{i}=\frac{\log _{2}\left(a b u n_{i+1}\right)-\log _{2}\left(a b u n_{i}\right)}{t_{i+1}-t_{i}}$. For comparison, we performed growth estimation using tools developed in previous studies (Korem et al., 2015; Brown et al., 2016; Emiola \& Oh, 2018; Gao $\& \mathrm{Li}, 2018)$. We added a time delay for the correlation coefficient between the experimental and growth estimates following the approach of the previous study (Korem et al., 2015). The time delay, which provided three or more combinations of the growth estimates and experimental growth rates and yielded the highest correlation coefficient, was accepted.

\section{Effect of normalization on the model}

We evaluated the effect of normalization on the parameter estimation by checking the difference between an estimated distribution and the true one. For the evaluation datasets, we generated continuous angles from the von Mises distribution. These angles were binned into discrete angles following the defined discrete length. We selected the location parameter from $-\pi,-\frac{\pi}{2}, 0$, and $\frac{\pi}{2}$ ; the concentration parameter from $0.1,0.4$, and 0.7 ; the discrete length to be segmented from 5 , $10,30,120,600$, and 1,000; and the average coverage depth to be observed from $0.5 \times 1.0 \times$, $2.0 \times, 4.0 \times, 8.0 \times$, and $16.0 \times$. Next, we fitted the unnormalized and normalized von Mises distribution-based models to the simulated data. Finally, we evaluated the error of the estimated distribution using the Kullback-Leibler divergence (Kullback \& Leibler, 1951). When evaluating the parameter of interest, the other parameters were fixed to 0 for the location parameter, 0.7 for the concentration parameter, 30 for the discrete length, and 16 for the average coverage depth. For the distribution with the true parameter, we set 10,000 as the discrete length. Calculations were performed 10 times with different seeds for each parameter set. This part of the procedure was performed using Scipy (Virtanen et al., 2019). We introduced a constraint $c_{\omega}$ to normalize the continuous circular distribution, i.e., $P_{\text {discrete }}\left(\theta_{n} \mid \omega\right)=\frac{1}{c_{\omega}} P_{\text {continuous }}(\theta \mid \omega)$ for discrete circular data $\theta_{n}(n=1,2, \ldots, N)$ and the parameter set $\omega$. For the von Mises and wrapped Cauchy 
388 distributions, we calculated the sum of the likelihood directly as $\log \left(c_{\omega}\right)=\log \left(\sum_{n=1}^{N} P\left(\theta_{n} \mid \omega\right)\right)$

389

390

391

392

393

394

395

396

397

398

399

400

401

402

403

404

405

406

407

408

409

410

411

412

413

414

415

416

417

418

419

420

421

422

423

424

425

because we could not have a closed-form equation. For the cardioid distribution, it was

formulated as $c_{\omega}=\sum_{n=1}^{N} \frac{1}{2 \pi}\left(1+2 \rho \cos \left(\theta_{n}-\mu\right)\right)=\frac{N}{2 \pi}$ owing to $\sum_{n=1}^{N} \cos (\theta)=0$. For the linear cardioid distribution, it was formulated as $c_{\omega}=\sum_{n=1}^{N} \frac{1}{2 \pi}\left(1+2 \rho\left(|| \theta-\mu|-\pi|-\frac{\pi}{2}\right)\right)=\frac{N+2}{2 \pi}$. The rearrangement of the formulas was performed using Sympy (Meurer et al., 2016).

\section{Simulation of skewness after coverage depth sorting and investigation of the causes}

The cause of the skewed shapes appearing at both ends of the coverage depth after sorting required investigation. The probable phage and duplicate gene regions are considered to generate outliers and to form the skewed shape. Thus, the probable regions on the chromosomal sequence were annotated. To identify probable phage regions in the genome, we used PHAST (Zhou et al., 2011). For the duplicate gene regions, first, we used Prokka to predict the coding sequences (Seemann, 2014). Next, we mapped these predicted sequences to the genome sequence of Lactobacillus gasseri using Bowtie2 and annotated regions as overlapping if two or more hits were obtained. The parameter set of Bowtie2 was "-a --very-sensitive." Thereafter, we extracted the partial coverage depth from 1.0-1.4 Mnt regions in which annotated features did not exist. To obtain the odds ratio, 36 metagenomic sequences obtained from the previous study (Korem et al., 2015) were mapped, and the coverage depth was calculated via the method described above.

Next, the coverage depth for each sample was sorted, and the number of annotated bases in 5\% of the upper, lower, and total sequence was counted. We modeled the number $n$ to follow a binomial distribution with the total number of bases $N$ of the target sequence and the appearance probability $p$ as parameters: $n \sim \operatorname{Binomial}(N, p)$. The odds ratio was calculated as odds $=\frac{p}{1-p}$ from the appearance probability $p$. To estimate these parameters, we employed an MCMC algorithm with NUTS using PyStan. Four chains were utilized, and 20,000 iterations were performed, where the first 1,000 of these were discarded as warm-ups. The posterior distribution of the EAP was used as the representative.

\section{Evaluation of robustness in terms of coverage depth using culture dataset}

To investigate the robustness of our proposed method, we compared the growth estimates calculated using a sufficient amount of reads and those using rarefied reads. To evaluate coverage depth dynamics from a single origin, we first used $L$. gasser $i$ WGS samples with an average of more than $20 \times$ coverage $(n=20)$. After confirming that many variations occur at lower than 5.0 $\times$ coverage, we selected Escherichia coli, Enterococcus faecalis, or L. gasseri WGSs reads with more than $5 \times$ coverage from the dataset by Korem and colleagues. To evaluate multiple origins of replication data, the WGS of $S$. solfataricus was used. We randomly sampled reads from the FASTQ files using seqtk $(\mathrm{Li}, 2013)$ such that the average coverage depth would 
426 be $0.001 \times, 0.005 \times, 0.01 \times, 0.05 \times, 0.1 \times, 0.5 \times, 1 \times$, or $5 \times$. In the first evaluation of a replication 427 dataset from a single origin, we additionally sampled $10 \times$ and $20 \times$ coverage. In the evaluation of

428

429

430

431

432

433

434

435

436

437

438

439

440

441

442

443

444

445

446

447

448

449

450

451

452

453

454

455

456

457

458

459

460

461

462

463

464 multiple origins, we additionally sampled $100 \times$ coverage. The pPTR, wPTR, and mwPTR were calculated using the rarefied reads and compared with those obtained from $20 \times$ and the full coverage depth. For the $S$. solfataricus dataset, we specified the number of components as three and performed estimation via the optimizing mode using 30 different seeds. We selected representative results with the highest likelihood and compared pPTR and mwPTR with those corresponding to no modifications. As DEMIC cannot work with a single genome sequence even if it is complete, we used a genome sequence obtained by co-assembling all of the reads using MEGAHIT with the default parameter set (Li et al., 2015). We utilized the default parameter set for PTRC, DEMIC, bPTR, iRep, and GRiD. Finally, we calculated the error rates as

$$
\left|\frac{\text { Estimate }_{\text {modified }}-\text { Estimate }_{\text {reference }}}{\text { Estimate }_{\text {reference }}}\right|
$$

The results from original WGS reads were used for reference estimates. To validate the error rate, we defined $15 \%$ as a threshold, as was done in a previous study (Brown et al., 2016).

\section{Evaluation of robustness in terms of coverage depth using metagenomics reads}

To evaluate the robustness using metagenomic datasets, we employed the inflammatory bowel disease (IBD) dataset from a previous study (Franzosa et al., 2018). We searched for combinations of species and WGSs with an average cover depth of $20 \times$ or more. In order for the first screening to satisfy the scope of the method, we used Kraken2 (Wood, Lu \& Langmead, 2019) and Bracken (Lu et al., 2017) with the default parameters; the objective of this was to count the number of reads to be assigned to the genome sequences. As a collection of complete chromosomal sequences, we constructed a database with species-level resolution (see Complete genome sequence database section). Based on the taxonomic profile, the combinations with more than $0.1 \times$ coverage depth were selected as candidates $(n=16,413$ combinations from 220 WGS samples) in the first screening. Next, we aligned the WGS reads of the datasets to the database using Bowtie2 and calculated the coverage depth using SAMtools. After applying the moving median filter and outlier elimination, we calculated the average coverage depth. If the combination had more than $20.0 \times$ average coverage and passed the first screening, we concluded that the combination was eligible to serve as an evaluation target $(n=676)$. For the targets, we extracted paired-end aligned reads using SAMtools with the "view -f 2" option for PTRC. This procedure was required because the GEM-mapper, which is used in PTRC, did not allow singletons to be aligned. After that, we rarefied the reads using SAMtools with the "view -s" option and converted the alignment result into a FASTQ file for the input. For our method, we counted the coverage depth from the alignment result using SAMtools. After applying each method, we calculated the error rate following equation (5). We used PTRC as a benchmark method, as it provided the most stable estimation with low coverage in the culture WGS dataset. 


\section{Evaluation of robustness in terms of mutation rate}

467

468

469

470

471

472

473

474

475

476

477

478

479

480

481

482

483

484

485

486

487

488

489

490

491

492

493

494

495

496

497

498

499

500

501

502

503

504

The robustness of our method in terms of the mutation rate with a single replication origin was assessed by evaluating how much of the value was maintained when the reference genome was used for mutation estimation. The reference genome sequence was mutated in three ways. The first involved nucleotide-level mutation at random positions in the genome sequence. Based on the length of the genome sequence, every $5 \%$ portion, ranging from 5 to $30 \%$ of the nucleotides, was randomly selected and mutated to an ambiguous nucleotide $\mathrm{N}$. The second way involved block-level mutation at random positions in the genome sequence. We used msbar in EMBOSS to create block-level mutated sequences (Rice, Longden \& Bleasby, 2000). Based on the length of the genome sequence, every $5 \%$ portion, ranging from 5 to $50 \%$ of the nucleotides, was randomly mutated. We used 5,000 nt as the block size. The third way involved mutation at the block level at a specific region in the genome sequence. We randomly selected the position to be mutated. The size of the region was determined based on the sequence length. After that, every $5 \%$ portion, ranging from 5 to $30 \%$ of the nucleotides, was mutated to an ambiguous nucleotide $\mathrm{N}$. As the positional relationship of the contig sequence was unknown, the evaluation of DEMIC in this regard was not performed. The first and third mutations were performed using in-house scripts with Biopython. After constructing pseudo-mutated genome sequences, we estimated and compared the growth following the above procedure. We assessed the robustness of the estimation with multiple replication origins, mutating the $S$. solfataricus genome sequence using the first and second methods. The estimations and evaluations for multiple origins of replication were performed in the same manner as the robustness evaluation with low coverage depth.

\section{Evaluation of robustness in terms of peak noise}

To investigate the influence of peak noise, we generated an artificial dataset. We used the shortread sequence of E. faecalis, L. gasseri, and S. solfataricus published previously (Korem et al., 2015; Payne et al., 2018). Firstly, a 100 bp region was randomly selected from a reference genome sequence using the seqkit sample command. The sequence was copied every 10 times from 10 to 100 times and added to the FASTQ files. We set 93 as the quality score. Using the mixed file, we calculated the coverage depth and estimated the growth in optimization mode following the procedure described above. For filtering, the top $1 \%$ of the depth was removed. Following this step, we computed the error rate using equation (5) and compared the results with the noiseless results. The evaluation for multiple replication origins was performed in the same manner as the evaluation for low coverage depth. Secondly, we randomly selected 1,000 bp regions from the reference genome sequence every 10 regions from 10 to 100 regions. This length was determined based on the average gene length of prokaryotes. These sequences were mixed with the WGS such that the coverage depth amounted to $20 \times, 40 \times, 60 \times, 80 \times$, and $100 \times$ in each region. Finally, we evaluated the error of the estimates following the same procedure. 


\section{Evaluation of robustness in terms of sample size}

507 To assess the effect of the sample size on the estimates, partial sample sets were generated from 508 the full sample set, and the results were compared. We used the short-read sequences of E. coli,

509 E. faecalis, and L. gasseri published previously (Korem et al., 2015). The partial set was

510 configured to include 1, 4, 8, 12, 16, and 20 samples. Each set was distributed in a manner that

511 avoided duplication of the same sample. Except for the sample set with only one sample, the sets

512 were constructed to contain each sample at least 10 times. For each sample set, preprocessing

513 and inference were conducted according to the above procedure, and the error rate was

514 calculated in comparison with the results obtained when all of the samples were used

515 simultaneously.

516

517

\section{Skewness in Watson and Crick strands}

519

To count the coverage depth in Watson strands and Crick strands, we used the SAMtools view command with the "-f" option set to 0 for the Watson strand and 16 for the Crick strand. This was done after mapping reads to the template genome sequence. The procedures that followed were the same for both strands. Finally, the highest log-likelihood in 30 independent trials was used as a representative estimate.

524

525

\section{Growth estimation of species with multiple replication origins}

We used the von Mises distribution for mixing because it has an intermediate degree of density around the mode and an open range of concentration parameters; i.e., $\kappa>0$. Both genomic and short-read sequences were obtained according to the procedures described previously (Ausiannikava et al., 2018; Payne et al., 2018). The coverage depth was calculated according to the above-mentioned procedure. As the deletion was confirmed in the genome sequence of $S$. solfataricus by the Integrative Genomics Viewer (Robinson et al., 2011), we deleted regions from 1,443,200 nt to $1,485,069 \mathrm{nt}$ on CP011055, from 1,443,192 nt to 1,485,075 nt on CP011056, and from 1,443,197 nt to $1,485,072$ nt on CP011057. We used Dfast to annotate cdc6 in the genome sequences (Tanizawa, Fujisawa \& Nakamura, 2018). The number of components $M$ was determined by using AIC and Widely Applicable Information Criterion (WAIC) as the mixture model was a singular model, and there was a possibility that a decision based only on AIC could produce incorrect results. The MCMC algorithm was applied to the constructed model, and WAIC was calculated from the log-likelihood (Watanabe, 2010; Gelman et al., 2013). The calculation was performed using an in-house Stan script and CmdStan with the threading option. The sampling was performed 1,500 times on a single chain, where the first 1,000 samplings were excluded as warm-ups. We fitted the model to the data assuming a number from 1 to 4 for the distribution. To calculate the AIC, we used the EAP of the posterior distribution to represent the log-likelihood. 
Growth rate estimation for infected Citrobacter rodentium

548 WGS reads of mice fecal samples from the original growth dynamics analysis study were used (Korem et al., 2015). These sequences were aligned to a complete genome sequence database (see Complete genome sequence database section) to validate the applicability of using multiple reference genome sequences using Bowtie 2 with the "--very-sensitive" option. After that, we counted the coverage depth by SAMtools. After extracting the coverage of the $C$. rodentium chromosome sequence, we cleaned and compressed the coverage using the moving median filter after removing the top $1 \%$. Finally, we fitted the von Mises model to the coverage. The pPTRs were compared via Welch's $t$-test.

556

557

\section{Complete genome sequence database}

559

The Genome Taxonomy Database version 89.0 was used to control the fineness of the taxonomy on the species level (Parks et al., 2018). For each species, when the representative species had a complete genome, it was used. When it did not, the sequence with the highest CheckM completeness score (Parks et al., 2015) and largest genome size was used. Species without complete genome sequences or those with multiple chromosome sequences were excluded. Mobile genetic elements were excluded from the database by checking the sequence label using seqkit; we filtered out the sequences labeled "plasmid," "Plasmid," "phage," "chromid," "pMLa," and "Linear."

\section{Growth estimate evaluation on metagenomic dataset}

The growth dynamics were estimated at species-level resolution. We filtered low-quality reads in WGS via Trimmomatic and then removed human-derived reads by aligning them to the GRCh38 reference human genomic sequence using Bowtie2. We used "SLIDINGWINDOW:4:15 MIN LEN:36" as a parameter in Trimmomatic. After quality control, we aligned qualified metagenomic reads with the complete genome sequence database using Bowtie 2 with the "-very-fast" option. After extracting the alignment results of the target reference sequence, we counted the coverage depth. As the metagenomic sequences were not clean compared to the culture datasets, we performed additional filtering as described in the coverage depth calculation method. After preprocessing was completed, we fitted the model to the coverage depth of each sequence via the optimizing mode. For the estimations, we selected samples with greater than or equal to $0.0001 \%$ relative abundance of the taxon and greater than $0.01 \times$ average coverage depth. We used Kraken2 and Bracken with the complete genome sequence database to estimate the relative abundance of the species. After filtering out the ultra-low coverage depth samples, we excluded the samples that might not achieve random sampling from the chromosomal DNA sequence. This is because the estimates of these samples would have an error. To detect the 
585 invalid samples, we focused on the difference of actual zero coverage fraction $f$ and a theoretical 586 score $\hat{f}$ based on the Lander-Waterman theory (Supplementary Text S4). This theoretical score 587 can be obtained as $\hat{f} \approx \exp (-a)$, where $a$ denotes the average coverage depth. For samples with 588 average coverage less than 5.0×, we excluded samples with log-scale fractions greater than 0.56 589 times the theoretical score. Assuming there to be uneliminated noise coverage depth, samples 590 with estimated PTRs greater than or equal to 3.0 were excluded. Welch's $t$-test for independent 591 groups was used to examine the differences between the growth estimates, and Hedges' $g$ was 592 used to evaluate the effect size for the two groups.

593

594

595

596

597

598

599

600

601

602

603

604

605

\section{Software}

We implemented the statistical model using Stan (Carpenter et al., 2017). Wrapped by Python scripts, this model is available for use in the command-line environment. This package also contains a moving median filter, a visualizer, a statistics profiler based on directional statistics, an information criterion calculator with estimated results, an asymmetric test calculator using Pewsey's method, and other utilities required to analyze the coverage depth over replicon. Other software versions are summarized in Supplementary Table 1. Our package for growth estimation is available from https://github.com/TaskeHAMANO/SPHERE. This software was implemented using Python3 ( $\geq 3.6$ ) and Stan. The wrapper software used in this study for PTRC, DEMIC, and GRiD is available from https://github.com/TaskeHAMANO/PTRC-in-cwl,

606 https://github.com/TaskeHAMANO/DEMIC-in-cwl, and

607 https://github.com/TaskeHAMANO/GRiD-in-cwl, respectively. This software is distributed under the BSD-3-Clause license. The wrapped software of msbar in EMBOSS is available from https://github.com/TaskeHAMANO/msbar-in-cwl. This software is distributed under the GPL3.0 license. These wrapper scripts were implemented using the Common Workflow Language

612

\section{Availability of data and material}

614 The WGS data of time-series-cultured E. coli, E. faecalis, L. gasseri, S. solfataricus, and $H$. volcanii are available from BioProject (PRJEB9718, PRJNA250819, PRJNA250820,

616 PRJNA250827, PRJNA346830, PRJNA250832, PRJNA250833, and PRJNA422812). The

617 genome sequences of E. coli NMC3722, E. faecalis ATCC 29212, L. gasseri ATCC33323, S.

618 solfataricus SULA, SARC-B, SARC-C, USLG, SARC-H, SARC-I, and $H$. volcanii DS2 are

619 available from GenBank and RefSeq (CP011495, CP008816, NC_008530, CP011057, 620 CP011055, CP011056, CP033235, CP033236, CP033237, and NC_013967). The genome sequence of $H$. volcanii $\mathrm{H} 26$ was modified from DS2 as previously described (Hawkins et al., 2013). The genome and metagenome sequences used in the cohort studies analysis are listed in

623 Supplementary Table 2 . The final chromosome sequences we used to construct the genome 624 sequence database are listed in Supplementary Table 3. 


\section{Results}

\section{Creating an artificial coverage depth}

629 We constructed a statistical model for coverage depth dynamics based on circular distributions.

630 To validate our model visually, we generated an artificial coverage depth using the above-

631 mentioned circular distributions (Supplementary Text S5). The generated coverage depth

632 reproduced high variance and concentration at the replication origin, expressed as the location

633 parameter of the circular distribution. Interestingly, when sorted, this artificial coverage depth

634 showed a distorted trend in both the upper and lower orders regardless of the circular distribution

635 type (Fig. 1a). The same shape was visualized previously (Brown et al., 2016), wherein it was

636 stated that this shape was formed by a specific sequence feature, such as a phage. However, our

637 model generated artificial depths from smooth probability trends and did not include any

638 artificial noise. To investigate in detail the cause of the distorted regions seen at both ends,

639 potential prophage sequences and duplicate genes in the genome sequence of Lactobacillus

640 gasseri were analyzed. Among them, only the intact prophage region was abundant at the lower

641 end (Supplementary Table 4). Moreover, a similar distorted structure was reproduced on a partial

642 genome sequence that did not contain suspicious regions (Fig. 1b). We evaluated the best model

643 for this distribution and used it to determine the threshold for outlier removal (Supplementary

644 Table 5; Supplementary Text S3).

645

646

647 Performance evaluation with experimental growth rates

648 Using a statistical model based on a circular distribution, we first evaluated the model's accuracy 649 by estimating the correlation between the computational and experimental growth rates, as had 650 been done previously (Korem et al., 2015). We estimated the coverage depth by mapping the 651 WGS reads to the genome sequence and counting the coverage depth (Fig. 2a). After reducing 652 the variance, outliers, and data size with a moving median filter, the proposed model was fitted 653 to the cleaned coverage depths (Fig. 2b). To evaluate the accuracy of the method, we used the 654 WGSs of the three species (E. coli, E. faecalis, and L. gasseri) previously obtained from culture 655 experiments under aerobic and anaerobic conditions (Korem et al., 2015). These data were 656 accompanied by $\mathrm{CFU} / \mathrm{ml}$ or OD in time series for evaluation. As substantiated in the previous 657 study, we observed a high correlation coefficient between the growth estimates and experimental 658 growth rates (Fig. 2c; Supplementary Fig. 2ab). Regardless of the culture state, E. coli and $E$.

659 faecalis exhibited high degrees of correlation, without requiring time delay adjustments $(\mathrm{r} \geq 0.5)$.

660 In contrast, $L$. gasseri required a time delay adjustment of 90-120 minutes. Our growth estimates

661 yielded correlation coefficients equivalent to those obtained using the previous methods, with 662 experimental growth rates of both 60 minutes (Supplementary Fig. 2c) and 30 minutes

663 (Supplementary Fig. 2d). Our estimates were correlated with the temporal growth based on the 664 relative abundance $(r=0.76 \pm 0.04, n=4$, each with 10 timepoints; Supplementary Fig. 2e) 
665 previously obtained (Korem et al., 2015) even when the samples originated from mixed cultures

666

667

668

669

670

671

672

673

674

675

676

677

678

679

680

681

682

683

684

685

686

687

688

689

690

691

692

693

694

695

696

697

698

699

700

701

702

703

704

with multiple intestinal species.

Secondly, we tuned the parameters of interest. The window and stride size of the moving median filter were optimized to $100 \mathrm{bp}$ by comparing the growth estimates with the experimental growth rates (Supplementary Figs. 3 and 4; Supplementary Texts S5 and S6). Our pipeline, which used a sequence aligner that did not take circular structures into account, confirmed that the decrease in coverage at both edges, termed the edge effect in a previous study (Brown et al., 2016), exerted only a small effect on the estimation (Supplementary Fig. 5; Supplementary Text S7). Our method performed well regarding memory usage and computation time with the exception of the Jones-Pewsey distribution-based model (Supplementary Fig. 6).

Finally, we evaluated the applicability of the method using artificial datasets. When applied to modified datasets from culture experiments, most methods, including those used in previous studies, have performed adequately with low coverage depth WGSs until at least $0.5 \times$, whereas our growth estimates remained stable even at coverage depths of $0.01 \times$ (Fig. 3a and Supplementary Fig. 7a). According to the bPTR in the coverage depth mode, the error percentage was not correlated with the number of reads. Although GRiD seemed to maintain a low error rate from $0.005 \times$ to $0.01 \times$ coverage depth, the number of samples with growth estimated as 1.0 increased $(0.005 \times: 10 / 20,0.01 \times: 17 / 20)$. As DEMIC requires multiple contigs for estimation and thus is not applicable to a single template genome sequence even when the sequence is complete, we performed the evaluation using assembled genome sequences. Moreover, this approach cannot be applied to low coverage less than or equal to $1.0 \times$ coverage. Given the culture dataset results, we also performed the evaluation on metagenomic reads and confirmed that the error rate was less than $15 \%$ on average until $0.01 \times$ coverage (Fig. 3b). Our method was also stable for mutations at both the 5,000 bp block and single nucleotide levels (Supplementary Figs. $7 \mathrm{~b}$ and c). Because the approach uses the entire sequence structure, the results obtained from a sequence mutated on a single specific region deviated from those of fulllength sequences (Supplementary Fig. 7d). To evaluate the effect in human intestine WGS reads, we quantified the deletion size on the chromosome sequences in a metagenomic dataset (Supplementary Fig. 8; Supplementary Text S8). Our estimates were as stable as those generated using the previous methods when a single peak noise was contaminated (Supplementary Fig. 7e). With more noise, although the error rate of our estimates remained less than $15 \%$ on average, some samples showed substantial error as the amount of artificial noise increased

(Supplementary Fig. 7f). To address this, we investigated the relationship between the error rate and the zero coverage fraction (Lander \& Waterman, 1988; Roach, 1995) and determined the threshold to exclude invalid samples with an error rate of more than $15 \%$. As a result, we detected the noise-contaminating coverage samples with a recall score of 0.81 (Supplementary Texts S4 and S9; Supplementary Figs. 9 and 10; Supplementary Table 6). Finally, the number of samples was related to the variation, but the effect was not substantial compared with those of the other factors (Supplementary Fig. $7 \mathrm{~g}$ ).

Peer) reviewing PDF | (2019:10:42075:3:0:NEW 6 Feb 2020) 


\section{Performance evaluation using in vivo dataset}

708

To evaluate the accuracy, we compared the growth estimates with the known growth dynamics

709 using previous datasets. For the in vivo sample setting, we tuned the window size of the moving median filter based on the coefficient of variance and concluded that $100 \mathrm{nt}$ was the best

710 (Supplementary Fig. 11). First, we checked the reproducibility of the growth estimates using $C$. rodentium-infected mice fecal samples. As was also reported previously (Korem et al., 2015), tir mutant $C$. rodentium had a higher pPTR than the wild-type (WT) strain (Supplementary Fig. 12; $p$-value by Welch's $t$-test between WT and mutant on days 6-9: $8.72 \times 10^{-5}, \mathrm{n}_{\mathrm{WT}}=12, \mathrm{n}_{\Delta t i r}=12$ ). Second, we evaluated the growth dynamics in the fecal microbiome in IBD patients (Franzosa et al., 2018). When we compared the estimates between Crohn's disease subjects and healthy volunteers, we reproduced the significant high growth estimates of Eggerthella lenta in the patients ( $p$-value: $1.26 \times 10^{-7}$, Hedges' $g=-1.21, \mathrm{n}_{\text {healthy }}=42, \mathrm{n}_{\text {Crohn }}=49$ ). Although this difference was limited to the remission and active patients in the small sample size dataset used in the previous study (Korem et al., 2015), we observed this difference in the large cohort dataset even by PTRC ( $p$-value: $1.31 \times 10^{-2}$, Hedge's $g=-0.57, \mathrm{n}_{\text {healthy }}=35, \mathrm{n}_{\text {Crohn }}=57$ ). Finally, we confirmed the growth dynamics of Bifidobacterium breve and Bifidobacterium adolescentis in the neonates and their mothers fecal microbiome cohort (Bäckhed et al., 2015). It is well known that $B$. breve is abundant in infant guts, whereas $B$. adolescentis is abundant in adult guts.

725 Moreover, a previous experimental study demonstrated that $B$. breve grows well in a medium containing formula based on soy, milk, or casein hydrolysate. These biological signals were also

727 reproduced in the estimates obtained using our model (Supplementary Fig. 13; Supplementary Table 7).

728

729

\section{Shape, peakedness, and skewness of coverage depth}

731

732 As an additional application of our model, we investigated the shape of the coverage depth by comparing the kinds of circular distributions(Supplementary Tables 8 and 9). In a comparison of the fitness of multiple models, the Jones-Pewsey distribution model exhibited the highest fitness among the vanilla models (those without argument transformation) on average. The shape parameter of the Jones-Pewsey distribution in the datasets of Korem et al, 2015. changes considerably with time (Supplementary Fig. 14a). For example, in the E. faecalis dataset, the distribution was dense around the replication origin in the first phase; however, it gradually dispersed over time. In contrast, the trend was reversed in the anaerobically cultured L. gasseri.

To evaluate the coverage depth concentration phenomenon further, we implemented the InvSE von Mises distribution model. Along with the Jones-Pewsey distribution model, the peakedness parameter of the InvSE von Mises distribution changed considerably with time (Supplementary Fig. 14b). Comparing the model with the vanilla von Mises-based model, the InvSE von Mises-based model exhibits a lower AIC and BIC (Supplementary Table 8). As in the

744 Jones-Pewsey model case, the peakedness was initially high. However, in E. faecalis, it became 
745 lower later on (Fig. 4). These parameters in the Jones-Pewsey and InvSE von Mises distributions

746 showed high correlation coefficients, but their trends were not identical $(\min \mathrm{r}=-0.793, \mathrm{p}=$

$7472.93 \times 10^{-7}$; Supplementary Fig. 15).

748 Next, we evaluated the symmetricity of replication. Although several methods that

749 extend circular probability densities toward asymmetricity have been described (Batschelet,

750 1981; Pewsey, 2002; Abe, Pewsey \& Fujisawa, 2013), a few requirements must be satisfied to

751 adapt to replication dynamics. Therefore, the InvMIAE von Mises distribution-based model was

752 used in this study. First, we evaluated the robustness of the asymmetric extended method

753 (Supplementary Text S10; Supplementary Fig. 16). As a result, we concluded that this extension

754 was not suitable for use with the short-read sequences that were largely mutated from the

755 template genome sequence. We therefore determined the applicability of the dataset by

756 estimating the mutation rate from the frequency of the zero-coverage depth using a zero-inflated

757 model (Supplementary Text S11). The results indicated that the E. faecalis dataset did not satisfy

758 the criteria (Supplementary Fig. 17a). Finally, we fitted the model to the actual coverage depth.

759 The skewness parameter had a low variance with time and was nearly 0 except for the E. faecalis

760 data (Supplementary Fig. 14b). Although E. faecalis showed high skewness, the InvMIAE model

761 fitted 0 or outlier coverage depths rather than the skewness of the whole sequence

762 (Supplementary Fig. 17b). Furthermore, we measured skewness using only Watson and Crick

763 strands. The skewness parameters showed strong correlations, and no specific skewness in any

764 specific strand was found (Supplementary Fig. 18). These results support the hypothesis that

765 coverage depth is symmetrical, contrary to our expectations.

766

767

768

769

\section{Extension of the model to multiple replication origins}

To demonstrate the extendibility of our method, we modeled the coverage depth behavior of

770

771 multiple replication origins, using a mixture of circular distributions. To validate our model, we applied it to WGS data of Sulfolobus solfataricus and Haloferax volcanii, which contain three

772 replication origins (Ausiannikava et al., 2018; Payne et al., 2018). Based on the AIC and WAIC,

773 we determined the number of components in both datasets to be three because this number

774 yielded the best score on average (Supplementary Table 10). Of the seven datasets, five matched

775 the true number of active replication origins. The estimated location parameters of S. solfataricus

776 were distributed close to $c d c 6$, which is a marker gene for the replication origin (the average error rate of the location parameter with respect to the marker gene is $6.55 \pm 4.48 \%, \mathrm{n}=6$ ) (Supplementary Figs. 19a-f) (Lundgren et al., 2004; Robinson et al., 2004). In contrast, although there is a distinct peak around $2 \mathrm{Mbp}, c d c 6$ is not evident in the $H$. volcanii genome sequence (Figs. 5a and b). We compared the weighted PTRs between the exponential growth and stationary phases. All of the origins in the exponential growth phase increased the estimates

782 (exponential growth phase: $3.59,3.18$, and 2.66; stationary phase: 1.64, 2.26, and 1.43). We also

783 checked the difference in the wPTR among multiple origins. Notably, the middle of the

784 replication origin position nearly coincided with the position at which the genomes were split by 
785 the ratio of wPTR (Figs. 5c and d; Supplementary Figs. 19g-1). As was done when the model 786 was applied for a single replication origin, the robustness of the estimates was evaluated using 787 the artificially modified dataset, which was an $S$. solfataricus dataset in this case. As a general 788 trend, the individually weighted PTRs were more sensitive to the modifications than the mean 789 weighted PTRs. When the number of reads was limited to $0.1 \times$ coverage depth on average, the 790 error of the estimates was less than 15\% at the median (Supplementary Fig. 19m). Although it 791 was more susceptible to noise than the model for a single origin of the replication origin, this

792 estimate was robust so long as the mutation rate was less than $7 \%$ at the point level or $4 \%$ at the 793 block level (Supplementary Figs. 19n and o). Our method avoided the effect of a single noise

794 region which increases coverage in the conserved region (Supplementary Fig. 19p).

795

796

797

798

799

800

801

802

803

804

805

806

807

808

\section{Discussion}

Here, we introduced a generative statistical model of coverage depth based on circular statistics and evaluated the estimated growth dynamics, replication trend, and differences in wPTR among multiple origins. In directional statistics, the simplest approach to expressing angular bias may be the use of the MRL. Although the MRL of the coverage depth was correlated with the experimental growth rate in the culture datasets, it is not as robust as estimates obtained via statistical models. This statistic can be easily calculated even with poor computational resources, but is not suitable for metagenomic datasets. Our proposed method was as accurate as the previous methods when compared with the experimental growth rates, and furthermore, it was robust against random mutations in the reference sequence and decreases in the coverage depth. Conversely, it was sensitive to a decrease in coverage depth due to mutations concentrated in a specific direction as well as to an increase in coverage depth due to conserved regions. In future research, it is expected that the rapid increase or decrease in coverage depth will be modeled to more accurately estimate the dynamics of the coverage depth. The simplest approach is not to use coverage depth in regions that are expected to be ineligible, as has been done in previous

\section{3} studies. However, this filtering approach alone does not provide a reasonable estimation for the proposed model as it also uses the absence of observation for parameter estimation. If a valid region $[a, b]$ can be assumed, ineligible regions could be excluded by normalizing the likelihood

816 from the metagenomic sequence, the average coverage depth and random sampling properties 817 must be examined, as was done here. Although we did not utilize it in this study, one of the 818 advantages of a GLM is its ability to incorporate covariate effects into the model. If one wants to 819 evaluate the relationship between the covariates $\mathbf{x}$ and pPTR, it is suitable to use a link function 820 for the concentration parameter. For example, when the von Mises distribution model is used, let $821 \beta$ be the coefficient of the covariates; then $\kappa=\exp \left(\beta_{0}+\beta_{1} x_{1}+\cdots\right)$ satisfies the requirement, i.e., $822 \kappa>0$. For a wrapped Cauchy distribution, the inverse logit function is appropriate to satisfy $8230<\rho<1$. 
824

825

826

827

828

829

830

831

832

833

834

835

836

837

838

839

840

841

842

843

844

845

846

847

848

849

850

851

852

853

854

855

856

857

858

859

860

861

862

863

We demonstrated the generation of artificial coverage depths using our statistical model. It was confirmed that the shape after sorting in ascending order was similar to the experimentally obtained replication profiles, which were presented previously (Brown et al., 2016). This shape did not depend on the type of circular distribution. These results demonstrate that the distorted shape could be generated not only by prophage sequences, strain variations, and highly conserved regions but also by the randomness of observation (DNA sequencing). When we evaluated this shape using actual data, we observed its appearance even in partial sequences that did not include these regions. This finding suggested that the shape is attributable not only to specific regions but also to the variance in observations, which is modeled by a multinomial distribution in our model. Filtering these parts out undoubtedly reduces the noise in the coverage depth.

In the evaluation using the in vivo dataset, we successfully confirmed consistency with previous studies. The species in the Bifidobacterium genus showed growth diversity when our method was evaluated using fecal WGS from infants and their mothers. Previous studies have revealed that Bifidobacterium adolescentis is abundant in adults and Bifidobacterium breve is abundant in infants (Turroni et al., 2012; Ruiz-Moyano et al., 2013; Kato et al., 2017); this trend was also reflected by the growth estimates. Although this finding was not reproduced by PTRC, previous studies have indicated that $B$. breve grows faster than other Bifidobacterium species in formula milk (Dubey \& Mistry, 1996) and human breast milk (Turroni et al., 2011). Since not all of the infants in the dataset had been weaned at the time of the study (Bäckhed et al., 2015), it is suggested that our method appropriately interpreted the dynamics.

When we compared the non-extended directional distributions for the replication trends, the Jones-Pewsey distribution exhibited the best fitness. This result implies that the additional parameter could contribute to the coverage depth dynamics that had been overlooked. The additional shape parameter implied that more reads were concentrated around the replication origin in the early stage of the exponential growth phase, except for L. gasseri in an anaerobic culture. We additionally applied the InvSE model to evaluate this phenomenon based on another quantification; this model reproduced the trend obtained using the Jones-Pewsey distribution model. We provide two possible explanations for the above phenomenon. The first is the effect of multiple replication forks. As the cell division phase is shorter than the genome replication phase in bacteria, the genome begins replication before finishing the current replication origin (Cooper \& Helmstetter, 1968; Bremer \& Churchward, 1977; Yoshikawa \& Wake, 1993; Wallden et al., 2016), allowing multiple rounds of replication to occur around the replication origin while rapid replication is occurring. Emiola and Oh also discussed the effect of multiple fork replication on the coverage depth (Emiola \& Oh, 2018). The second hypothesis is that, as the entire chromosome is not affected at the start of DNA replication, some DNA appears only around the replication origin. However, this concept does not explain the generation time of bacteria. Under laboratory conditions, DNA replication of E. coli is reportedly completed within approximately 30 minutes (Helmstetter \& Cooper, 1968). If the second hypothesis was valid, the additional coverage depth concentration around the origin should be finished within 30 minutes

Peer] reviewing PDF | (2019:10:42075:3:0:NEW 6 Feb 2020) 
864 from the beginning of the culture. However, the degree of density remained low for an hour in

865

866

867

868

869

870

871

872

873

874

875

876

877

878

879

880

881

882

883

884

885

886

887

888

889

890

891

892

893

894

895

896

897

898

899

900

901

902

903 our study. The trend observed in the anaerobic cultured L. gasseri was the opposite of what was seen in the others; however, it is worth noting that L. gasseri required 90-120 minutes of adjustment to have a sufficient correlation between the experimental growth rate and estimated growth dynamics. This suggests that both the activation of DNA replication and cell division are required to decrease the degree of density. Accordingly, we inferred that the degree of density and peakedness may indicate the activity of multiple replication forks. In contrast, the skewness parameters in E. coli and L. gasseri did not change dynamically during the experimental duration. Additionally, we confirmed the presence of a strong correlation between the skewness of the Watson and Crick strands, implying that the amount of DNA remains symmetric between the Watson and Crick strands as well as between the leading and lagging strands.

In addition to the application to microbes with a single replication origin, we extended the model's application to microbes with multiple origins in a single chromosome. One interesting finding was the difference in wPTR among multiple origins. From the relationship between the intermediate position of the origins and the split position of the chromosome sequence based on wPTR, the efficiency, in terms of the activity of the origins, was quantitatively confirmed. If only a single replication origin was active in a chromosome, considerable time could be required for whole-genome replication, which would be a disadvantage for survival. By properly activating the origins at a distance, replication may be efficiently completed. However, our results indicated that not all replication origins exhibit similar activity. There are various characteristics that cause the activity to differ, such as (a)synchronous initiation (Lundgren et al., 2004), replication fork speed (Elshenawy et al., 2015), and so on. Therefore, the mechanism underlying the differences observed for each replication origin must be clarified, and the characteristics of neighboring genes must be investigated.

The current study was affected by certain limitations. First, the proposed method requires circular genome sequences for accurate estimation. As several methods involving contig or scaffold-level sequences have already been proposed for estimating the quasi-growth of bacteria (Brown et al., 2016; Emiola \& Oh, 2018; Gao \& Li, 2018), it is recommended that these methods be properly used depending on the accuracy requirements. It is difficult to detect trends in the amount of DNA other than the coverage depth bias or to estimate the bias in chromosomes with multiple replication origins using these methods. We consider our method to be appropriate for data analyses related to detailed replication profiles.

Second, the taxonomic resolution is limited to the species level in our method, on account of the first limitation. When the growth estimates of a reference strain were calculated using metagenome samples containing different but closely related strains, their growth dynamics were found to be different, indicating that the pPTR distributions may be mixed. This difficulty regarding the taxonomic resolution has yet to be solved via growth rate estimation, which may give rise to major challenges in environments such as soil, wherein many closely related species are contained because of empty niches and/or microstructures (Dumbrell et al., 2010; Thompson et al., 2017). However, this challenge may be less serious in environments devoid of close

Peer] reviewing PDF | (2019:10:42075:3:0:NEW 6 Feb 2020) 
904 relatives on account of the filling of niche space and/or strong selective pressure. The human 905 intestine likely corresponds to the latter case (Jeraldo et al., 2012; Li \& Ma, 2016; Thompson et

906 al., 2017) but may shift to the former case in situations in which the population is being

907 reconstructed because of an environmental change (Langenheder \& Szekely, 2011). This

908 resolution problem may be solved by constructing pan-genome sequences from metagenomic

909 reads and allocating coverage depth appropriately. Third, the evaluation scope of the extended

910 model is limited. Although we evaluated and eliminated the possibility of overfitting in our

911 dataset, we cannot deny the possibility that the dynamics of the peakedness and stability of the

912 skewness around the origin are specific to the three strains we used. External validations are

913 expected to confirm the variability of the peakedness or stability of the skewness over the growth

914 phase. Finally, in our method as well as all currently proposed methods for estimating bacterial

915 growth, the estimate itself is only a proxy of the growth rate. Theoretically, (p)PTR for a taxon $t$

916 in a sample $s$ is represented by $(p) P T R_{s, t}=2^{C_{t} / \tau_{s, t}}$, where $C_{t}$ is the replication period and $\tau_{s, t}$ is

917 the doubling time (Cooper \& Helmstetter, 1968; Bremer \& Churchward, 1977; Korem et al.,

918 2015). Our interest is in the doubling time, but the estimate is also influenced by the replication

919 period. This period may vary from species to species depending on the genome size and other

920 factors. Therefore, it is not appropriate to compare estimates between species. It is necessary to

921 analyze the effects of the replication period $C$ and to propose a method that yields a doubling

922 time that is comparable between species (Gibson et al., 2018).

923

\section{Conclusions}

925 We developed a probabilistic model based on circular statistics to model the coverage depth

926 behavior in DNA replication using WGS reads. This method was demonstrated to be robust for a

927 small number of reads $(\geq 0.01 \times)$. The probabilistic PTR from our model demonstrated a

928 significant correlation with the experimental growth rates in the culture dataset. In addition to

929 facilitating quantification of the ratio differences, this method enables detailed measurement of

930 DNA quantity changes by using circular distributions in the model. Moreover, by combining

931 multiple distributions, it became possible to estimate the growth of organisms with multiple

932 replication origins, such as archaea. Therefore, this method further extends the applicability of

933 growth estimation from fragmented reads. We expect that the growth estimation method

934 presented herein will help elucidate factors that have not yet been observed in studies of

935 microbiome formation.

936

937 Acknowledgements

938 We thank Ken Kurokawa, Hiroshi Mori, and Koichi Higashi for discussions regarding the

939 research design during the early stages of this work. We thank Yuya Nakamura for discussions

940 regarding the statistical modeling experiment and design of the linear cardioid model. We thank

941 Darzi Youssef for his review of the in vivo dataset section. We thank Anton Björk for his

942 essential advice related to the skewable asymmetric extended model. We thank Thorsten Allers 
943 for providing the $H$. volcanii genome sequence. We are grateful to the reviewers for their

944 numerous helpful comments, which helped us to improve this report.

945

946

947

948

949

950

951

952

953

954

955

956

957

958

959

960

961

962

963

964

965

966

967

968

969

970

971

972

973

974

975

976

977

978

979

980

981

982

983

984

985

986

987

988

989

990

991

992

993

994

995

\section{References}

Abe T, Pewsey A, Fujisawa N. 2013. Asymmetric distribution family on circumference with mode invariance. In: JFSAA. Osaka, 1.

Abe T, Pewsey A, Shimizu K. 2013. Extending circular distributions through transformation of argument. Annals of the Institute of Statistical Mathematics 65:833-858. DOI: 10.1007/s10463-012-0394-5.

Akiyama MT, Oshima T, Chumsakul O, Ishikawa S, Maki H. 2016. Replication fork progression is paused in two large chromosomal zones flanking the DNA replication origin in Escherichia coli. Genes to Cells 21:907-914. DOI: $10.1111 /$ gtc. 12388 .

Andersson AF, Pelve EA, Lindeberg S, Lundgren M, Nilsson P, Bernander R. 2010. Replication-biased genome organisation in the crenarchaeon Sulfolobus. BMC Genomics 11:454. DOI: 10.1186/1471-2164-11-454.

Ausiannikava D, Mitchell L, Marriott H, Smith V, Hawkins M, Makarova KS, Koonin E V, Nieduszynski CA, Allers T. 2018. Evolution of genome architecture in archaea: spontaneous generation of a new chromosome in Haloferax volcanii. Mol. Biol. Evol. 35:1855-1868. DOI: 10.1093/molbev/msy075.

Bäckhed F, Roswall J, Peng Y, Feng Q, Jia H, Kovatcheva-Datchary P, Li Y, Xia Y, Xie H, Zhong H, Khan MT, Zhang J, Li J, Xiao L, Al-Aama J, Zhang D, Lee YS, Kotowska D, Colding C, Tremaroli V, Yin Y, Bergman S, Xu X, Madsen L, Kristiansen K, Dahlgren J, Jun W. 2015. Dynamics and stabilization of the human gut microbiome during the first year of life. Cell Host and Microbe 17:690-703. DOI: 10.1016/j.chom.2015.04.004.

Batschelet E. 1981. Circular statistics in biology. Academic Press Inc.

Benjamini Y, Speed TP. 2012. Summarizing and correcting the GC content bias in high-throughput sequencing. Nucleic Acids Research 40:1-14. DOI: 10.1093/nar/gks001.

Bremer H, Churchward G. 1977. An examination of the Cooper-Helmstetter theory of DNA replication in bacteria and its underlying assumptions. Journal of Theoretical Biology 69:645-654. DOI: 10.1016/00225193(77)90373-3.

Brown CT, Olm MR, Thomas BC, Banfield JF. 2016. Measurement of bacterial replication rates in microbial communities. Nature Biotechnology 34:1256-1263. DOI: 10.1038/nbt.3704.

Carpenter B, Gelman A, Hoffman MD, Lee D, Goodrich B, Betancourt M, Brubaker M, Guo J, Li P, Riddell A. 2017. Stan: A probabilistic programming language. J. Stat. Softw. 76. DOI: 10.18637/jss.v076.i01.

Chen L, Brügger K, Skovgaard M, Redder P, She Q, Torarinsson E, Greve B, Awayez M, Zibat A, Klenk H-P, Garrett RA, Bru K, Redder P, Awayez M. 2005. The genome of Sulfolobus acidocaldarius, a model organism of the Crenarchaeota. J. Bacteriol. 187:4992-4999. DOI: 10.1128/JB.187.14.4992-4999.2005.

Cooper S, Helmstetter CE. 1968. Chromosome replication and the division of Escherichia coli B/r. Journal of Molecular Biology 31:519-540. DOI: 10.1016/0022-2836(68)90425-7.

Dowell M, Jarratt P. 1971. A modified regula falsi method for computing the root of an equation. BIT Numerical Mathematics 11:168-174. DOI: 10.1007/bf01934364.

Dubey UK, Mistry VV. 1996. Growth characteristics of bifidobacteria in infant formulas. Journal of Dairy Science 79:1146-1155. DOI: 10.3168/jds.s0022-0302(96)76468-8.

Dumbrell AJ, Nelson M, Helgason T, Dytham C, Fitter AH. 2010. Relative roles of niche and neutral processes in structuring a soil microbial community. ISME Journal 4:337-345. DOI: 10.1038/ismej.2009.122.

Elshenawy MM, Jergic S, Xu Z-QQ, Sobhy MA, Takahashi M, Oakley AJ, Dixon NE, Hamdan SM. 2015. Replisome speed determines the efficiency of the Tus-Ter replication termination barrier. Nature 525:394 398. DOI: $10.1038 /$ nature 14866 .

Emiola A, Oh J. 2018. High throughput in situ metagenomic measurement of bacterial replication at ultra-low sequencing coverage. Nature Communications 9:4956. DOI: 10.1038/s41467-018-07240-8.

Forsyth VS, Armbruster CE, Smith SN, Pirani A, Springman AC, Walters MS, Nielubowicz GR, Himpsl SD, Snitkin ES, Mobley HLTT. 2018. Rapid growth of uropathogenic Escherichia coli during human urinary tract infection. mBio 9:e00186-18. DOI: 10.1128/MBIO.00186-18.

Franzosa EA, Sirota-madi A, Avila-pacheco J, Fornelos N, Haiser HJ, Reinker S, Vatanen T, Hall AB, Mallick H, Mciver LJ, Sauk JS, Wilson RG, Stevens BW, Scott JM, Pierce K, Deik AA, Bullock K, Imhann F, Porter JA, Zhernakova A, Fu J, Weersma RK, Wijmenga C, Clish CB, Vlamakis H, Huttenhower C, Xavier RJ. 2018.

Peer] reviewing PDF | (2019:10:42075:3:0:NEW 6 Feb 2020) 
996

997

998

999

1000

1001

1002

1003

1004

1005

1006

1007

1008

1009

1010

1011

1012

1013

1014

1015

1016

1017

1018

1019

1020

1021

1022

1023

1024

1025

1026

1027

1028

1029

1030

1031

1032

1033

1034

1035

1036

1037

1038

1039

1040

1041

1042

1043

1044

1045

1046

1047

1048

1049

1050
Gut microbiome structure and metabolic activity in inflammatory bowel disease. Nature Microbiology 4:293305. DOI: 10.1038/s41564-018-0306-4.

Gao F. 2015. Bacteria may have multiple replication origins. Front. Microbiol. 6:1-4. DOI: 10.3389/fmicb.2015.00324.

Gao Y, Li H. 2018. Quantifying and comparing bacterial growth dynamics in multiple metagenomic samples. Nature Methods. DOI: 10.1038/s41592-018-0182-0.

Gao F, Zhang CT. 2008. Ori-Finder: A web-based system for finding oriCs in unannotated bacterial genomes. $B M C$ Bioinformatics 9:1-6. DOI: 10.1186/1471-2105-9-79.

Garrison E, Sirén J, Novak AM, Hickey G, Eizenga JM, Dawson ET, Jones W, Garg S, Markello C, Lin MF, Paten B, Durbin R. 2018. Variation graph toolkit improves read mapping by representing genetic variation in the reference. Nat. Biotechnol. 36:875-879. DOI: 10.1038/nbt.4227.

Gelman A. 2006. Prior distributions for variance parameters in hierarchical models. Bayesian Analysis 1:515-533. DOI: $10.1214 / 06-B A 117 A$.

Gelman A, Carlin J, Stern H, Dunson D, Vehtari A, Rubin D. 2013. Bayesian data analysis. CRC Press.

Gibson B, Wilson DJ, Feil E, Eyre-Walker A. 2018. The distribution of bacterial doubling times in the wild. Proceedings of the Royal Society B: Biological Sciences 285. DOI: 10.1098/rspb.2018.0789.

Hawkins M, Malla S, Blythe MJ, Nieduszynski CA, Allers T. 2013. Accelerated growth in the absence of DNA replication origins. Nature 503:544-547. DOI: 10.1038/nature12650.

Helmstetter CE, Cooper S. 1968. DNA synthesis during the division cycle of rapidly growing Escherichia coli B/r. Journal of Molecular Biology 31:507-518. DOI: 10.1016/0022-2836(68)90424-5.

Higashi K, Suzuki S, Kurosawa S, Mori H, Kurokawa K. 2018. Latent environment allocation of microbial community data. PLoS Comput. Biol. 14:e1006143. DOI: 10.1371/journal.pcbi.1006143.

Hildebrand F, Nguyen TLA, Brinkman B, Yunta RG, Cauwe B, Vandenabeele P, Liston A, Raes J. 2013. Inflammation-associated enterotypes, host genotype, cage and inter-individual effects drive gut microbiota variation in common laboratory mice. Genome Biology 14:R4. DOI: 10.1186/gb-2013-14-1-r4.

Hoffman MD, Gelman A. 2014. The No-U-Turn Sampler: Adaptively setting path lengths in Hamiltonian Monte Carlo. Journal of Machine Learning Research 15:1593-1623. DOI: abs/1111.4246.

Jeraldo P, Sipos M, Chia N, Brulc JM, Dhillon AS, Konkel ME, Larson CL, Nelson KE, Qu A, Schook LB, Yang F, White BA, Goldenfeld N. 2012. Quantification of the relative roles of niche and neutral processes in structuring gastrointestinal microbiomes. Proceedings of the National Academy of Sciences 109:9692-9698. DOI: $10.1073 /$ pnas.1206721109.

Jones MC, Pewsey A. 2005. A family of symmetric distributions on the circle. J. Am. Stat. Assoc. 100:1422-1428. DOI: $10.1198 / 016214505000000286$.

Kato H, Mori H, Maruyama F, Toyoda A, Oshima K, Endo R, Fuchu G, Miyakoshi M, Dozono A, Ohtsubo Y, Nagata Y, Hattori M, Fujiyama A, Kurokawa K, Tsuda M. 2015. Time-series metagenomic analysis reveals robustness of soil microbiome against chemical disturbance. DNA Research 22:413-424. DOI: 10.1093/dnares/dsv023.

Kato K, Odamaki T, Mitsuyama E, Sugahara H, Xiao J zhong, Osawa R. 2017. Age-related changes in the composition of gut bifidobacterium species. Current Microbiology 74:987-995. DOI: 10.1007/s00284-0171272-4.

Korem T, Zeevi D, Suez J, Weinberger A, Avnit-Sagi T, Pompan-Lotan M, Matot E, Jona G, Harmelin A, Cohen N, Sirota-Madi A, Thaiss CA, Pevsner-Fischer M, Sorek R, Xavier RJ, Elinav E, Segal E. 2015. Growth dynamics of gut microbiota in health and disease inferred from single metagenomic samples. Science 349:1101-1106. DOI: 10.1126/science.aac4812.

Kullback S, Leibler RA. 1951. On information and sufficiency. Ann. Math. Stat. 22:79-86. DOI: 10.1214/aoms/1177729694.

Lander ES, Waterman MS. 1988. Genomic mapping by fingerprinting random clones: A mathematical analysis. Genomics 2:231-239. DOI: 10.1016/0888-7543(88)90007-9.

Langenheder S, Szekely AJ. 2011. Species sorting and neutral processes are both important during the initial assembly of bacterial communities. Isme Journal 5:1086-1094. DOI: 10.1038/Ismej.2010.207.

Langmead B, Salzberg SL. 2012. Fast gapped-read alignment with Bowtie 2. Nat. Methods 9:357-359. DOI: 10.1038/nmeth. 1923 .

Leman AR, Noguchi E. 2013. The replication fork: Understanding the eukaryotic replication machinery and the challenges to genome duplication. Genes 4:1-32. DOI: 10.3390/genes4010001.

Li H. 2013.lh3/seqtk. Available at https://github.com/lh3/seqtk (accessed December 10, 2018). 
1051

1052

1053

1054

1055

1056

1057

1058

1059

1060

1061

1062

1063

1064

1065

1066

1067

1068

1069

1070

1071

1072

1073

1074

1075

1076

1077

1078

1079

1080

1081

1082

1083

1084

1085

1086

1087

1088

1089

1090

1091

1092

1093

1094

1095

1096

1097

1098

1099

1100

1101

1102

1103

1104

1105

Li H, Handsaker B, Wysoker A, Fennell T, Ruan J, Homer N, Marth G, Abecasis G, Durbin R. 2009. The sequence alignment/map format and SAMtools. Bioinformatics 25:2078-2079. DOI: 10.1093/bioinformatics/btp352.

Li D, Liu CM, Luo R, Sadakane K, Lam TW. 2015. MEGAHIT: An ultra-fast single-node solution for large and complex metagenomics assembly via succinct de Bruijn graph. Bioinformatics 31:1674-1676. DOI: 10.1093/bioinformatics/btv033.

Li L, Ma ZS. 2016. Testing the neutral theory of biodiversity with human microbiome datasets. Scientific Reports 6:1-10. DOI: 10.1038/srep31448.

Lu J, Breitwieser FP, Thielen P, Salzberg SL. 2017. Bracken: Estimating species abundance in metagenomics data. PeerJ 2017:1-17. DOI: 10.7717/peerj-cs.104.

Lundgren M, Andersson A, Chen L, Nilsson P, Bernander R. 2004. Three replication origins in Sulfolobus species: synchronous initiation of chromosome replication and asynchronous termination. Proceedings of the National Academy of Sciences of the United States of America 101:7046-51. DOI: 10.1073/pnas.0400656101.

Maduike NZ, Tehranchi AK, Wang JD, Kreuzer KN. 2014. Replication of the Escherichia coli chromosome in RNase HI-deficient cells: Multiple initiation regions and fork dynamics. Molecular Microbiology 91:39-56. DOI: $10.1111 / \mathrm{mmi} .12440$.

McCarthy S, Gradnigo J, Johnson T, Payne S, Lipzen A, Martin J, Schackwitz W, Moriyama E, Blum P, Julien G, Tyler J, Payne S, Anna L, Joel M, Wendy S, Etsuko M, Paul B. 2015. Complete genome sequence of sulfolobus solfataricus strain 98/2 and evolved derivatives. Genome Announc. 3:61779. DOI: 10.1128/genomeA.00549-15.

Meurer A, Smith CP, Paprocki M, Čertík O, Rocklin M, Kumar Am, Ivanov S, Moore JK, Singh S, Rathnayake T, Vig S, Granger BE, Muller RP, Bonazzi F, Gupta H, Vats S, Johansson F, Pedregosa F, Curry MJ, Saboo A, Fernando I, Kulal S, Cimrman R, Scopatz A, Kirpichev SB, Rocklin M, Kumar Am, Ivanov S, Moore JK, Singh S, Rathnayake T, Vig S, Granger BE, Muller RP, Bonazzi F, Gupta H, Vats S, Johansson F, Pedregosa F, Curry MJ, Terrel AR, Roučka Š, Saboo A, Fernando I, Kulal S, Cimrman R, Scopatz A. 2016. SymPy: Symbolic computing in Python. PeerJ Computer Science 3:e103. DOI: 10.7287/peerj.preprints.2083v2.

Milanese A, Mende DR, Paoli L, Salazar G, Ruscheweyh HJ, Cuenca M, Hingamp P, Alves R, Costea PI, Coelho LP, Schmidt TSB, Almeida A, Mitchell AL, Finn RD, Huerta-Cepas J, Bork P, Zeller G, Sunagawa S. 2019. Microbial abundance, activity and population genomic profiling with mOTUs2. Nature Communications 10. DOI: 10.1038/s41467-019-08844-4.

Ohbayashi R, Watanabe S, Ehira S, Kanesaki Y, Chibazakura T, Yoshikawa H. 2016. Diversification of DnaA dependency for DNA replication in cyanobacterial evolution. ISME Journal 10:1113-1121. DOI: 10.1038/ismej.2015.194.

Olm MR, Brown CT, Brooks B, Firek B, Baker R, Burstein D, Soenjoyo K, Thomas BC, Morowitz M, Banfield JF. 2017. Identical bacterial populations colonize premature infant gut, skin, \& oral microbiomes \& exhibit different in situ growth rates. Genome Research 27:601-612. DOI: 10.1101/gr.213256.116.

Parks DH, Chuvochina M, Waite DW, Rinke C, Skarshewski A, Chaumeil P-A, Hugenholtz P. 2018. A standardized bacterial taxonomy based on genome phylogeny substantially revises the tree of life. Nature Biotechnology 36:996-1004. DOI: 10.1038/nbt.4229.

Parks DH, Imelfort M, Skennerton CT, Hugenholtz P, Tyson GW. 2015. CheckM: Assessing the quality of microbial genomes recovered from isolates, single cells, and metagenomes. Genome Research 25:1043-1055. DOI: $10.1101 / \mathrm{gr} .186072 .114$.

Payne S, McCarthy S, Johnson T, North E, Blum P, Pattwell SS, Bath KG, Casey BJ, Ninan I, Lee FS, Mcewen BS, Pattwellab SS, Bathacd KG, Caseya BJ, Ninan I, Leea FS. 2018. Nonmutational mechanism of inheritance in the archaeon Sulfolobus solfataricus. Proceedings of the National Academy of Sciences 115:12271-12276. DOI: 10.1073 PNAS.

Pelve EA, Martens-Habbena W, Stahl DA, Bernander R. 2013. Mapping of active replication origins in vivo in thaum- and euryarchaeal replicons. Molecular Microbiology 90:538-550. DOI: 10.1111/mmi.12382.

Pewsey A. 2002. Testing circular symmetry. The Canadian Journal of Statistics 30:591-600. DOI: $10.2307 / 3316098$.

Pewsey A, Neuhäuser M, Ruxton GD. 2013. Circular statistics in R. Oxford University Press.

Retkute R, Hawkins M, Rudolph CJ, Nieduszynski CA. 2018. Modeling of DNA replication in rapidly growing bacteria with one and two replication origins. bioRxiv:1-22. DOI: 10.1101/354654.

Rice P, Longden L, Bleasby A. 2000. EMBOSS: The European molecular biology open software suite. Trends in Genetics 16:276-277. DOI: 10.1016/S0168-9525(00)02024-2.

Roach JC. 1995. Random subcloning. Genome Research 5:464-473. DOI: 10.1101/gr.5.5.464.

Peer) reviewing PDF | (2019:10:42075:3:0:NEW 6 Feb 2020) 
1106

1107

1108

1109

1110

1111

1112

1113

1114

1115

1116

1117

1118

1119

1120

1121

1122

1123

1124

1125

1126

1127

1128

1129

1130

1131

1132

1133

1134

1135

1136

1137

1138

1139

1140

1141

1142

1143

1144

1145

1146

1147

1148

1149

1150

1151

1152

1153

1154

1155

1156

1157

1158

1159

1160

1161
Robinson NP, Dionne I, Lundgren M, Marsh VL, Bernander R, Bell SD. 2004. Identification of two origins of replication in the single chromosome of the archaeon Sulfolobus solfataricus. Cell 116:25-38. DOI: 10.1016/S0092-8674(03)01034-1.

Robinson JT, Thorvaldsdóttir H, Winckler W, Guttman M, Lander ES, Getz G, Mesirov JP. 2011. Integrative genomics viewer. Nature Biotechnology 29:24-26. DOI: 10.1038/nbt.1754.

Rodriguez-Lopez AM, Jackson DA, Iborra F, Cox LS, Rodríguez-López AM, Jackson DA, Iborra F, Cox LS. 2002. Asymmetry of DNA replication fork progression in Werner's syndrome. Aging cell 1:30-39. DOI: 10.1046/j.1474-9728.2002.00002.x.

Ross MG, Russ C, Costello M, Hollinger A, Lennon NJ, Hegarty R, Nusbaum C, Jaffe DB. 2013. Characterizing and measuring bias in sequence data. Genome Biology 14. DOI: 10.1186/gb-2013-14-5-r51.

Rudolph CJ, Upton AL, Stockum A, Nieduszynski CA, Lloyd RG. 2013. Avoiding chromosome pathology when replication forks collide. Nature 500:608-611. DOI: 10.1038/nature12312.

Ruiz-Moyano S, Totten SM, Garrido DA, Smilowitz JT, Bruce German J, Lebrilla CB, Mills DA. 2013. Variation in consumption of human milk oligosaccharides by infant gut-associated strains of bifidobacterium breve. Applied and Environmental Microbiology 79:6040-6049. DOI: 10.1128/AEM.01843-13.

Seemann T. 2014. Prokka: Rapid prokaryotic genome annotation. Bioinformatics 30:2068-2069. DOI: 10.1093/bioinformatics/btu153.

Sernova N V., Gelfand MS. 2008. Identification of replication origins in prokaryotic genomes. Briefings in Bioinformatics 9:376-391. DOI: 10.1093/bib/bbn031.

Tanizawa Y, Fujisawa T, Nakamura Y. 2018. DFAST: A flexible prokaryotic genome annotation pipeline for faster genome publication. Bioinformatics 34:1037-1039. DOI: 10.1093/bioinformatics/btx713.

Thompson LR, Sanders JG, McDonald D, Amir A, Ladau J, Locey KJ, Prill RJ, Tripathi A, Gibbons SM, Ackermann G, Navas-Molina JA, Janssen S, Kopylova E, Vázquez-Baeza Y, González A, Morton JT, Mirarab S, Zech Xu Z, Jiang L, Haroon MF, Kanbar J, Zhu Q, Jin Song S, Kosciolek T, Bokulich NA, Lefler J, Brislawn CJ, Humphrey G, Owens SM, Hampton-Marcell J, Berg-Lyons D, McKenzie V, Fierer N, Fuhrman JA, Clauset A, Stevens RL, Shade A, Pollard KS, Goodwin KD, Jansson JK, Gilbert JA, Knight R, Rivera JLA, Al-Moosawi L, Alverdy J, Amato KR, Andras J, Angenent LT, Antonopoulos DA, Apprill A, Armitage D, Ballantine K, Bárta J, Baum JK, Berry A, Bhatnagar A, Bhatnagar M, Biddle JF, Bittner L, Boldgiv B, Bottos E, Boyer DM, Braun J, Brazelton W, Brearley FQ, Campbell AH, Caporaso JG, Cardona C, Carroll J, Cary SC, Casper BB, Charles TC, Chu H, Claar DC, Clark RG, Clayton JB, Clemente JC, Cochran A, Coleman ML, Collins G, Colwell RR, Contreras M, Crary BB, Creer S, Cristol DA, Crump BC, Cui D, Daly SE, Davalos L, Dawson RD, Defazio J, Delsuc F, Dionisi HM, Dominguez-Bello MG, Dowell R, Dubinsky EA, Dunn PO, Ercolini D, Espinoza RE, Ezenwa V, Fenner N, Findlay HS, Fleming ID, Fogliano V, Forsman A, Freeman C, Friedman ES, Galindo G, Garcia L, Garcia-Amado MA, Garshelis D, Gasser RB, Gerdts G, Gibson MK, Gifford I, Gill RT, Giray T, Gittel A, Golyshin P, Gong D, Grossart H-P, Guyton K, Haig S-J, Hale V, Hall RS, Hallam SJ, Handley KM, Hasan NA, Haydon SR, Hickman JE, Hidalgo G, Hofmockel KS, Hooker J, Hulth S, Hultman J, Hyde E, Ibáñez-Álamo JD, Jastrow JD, Jex AR, Johnson LS, Johnston ER, Joseph S, Jurburg SD, Jurelevicius D, Karlsson A, Karlsson R, Kauppinen S, Kellogg CTE, Kennedy SJ, Kerkhof LJ, King GM, Kling GW, Koehler A V., Krezalek M, Kueneman J, Lamendella R, Landon EM, Lane-deGraaf K, LaRoche J, Larsen P, Laverock B, Lax S, Lentino M, Levin II, Liancourt P, Liang W, Linz AM, Lipson DA, Liu Y, Lladser ME, Lozada M, Spirito CM, MacCormack WP, MacRae-Crerar A, Magris M, Martín-Platero AM, Martín-Vivaldi M, Martínez LM, Martínez-Bueno M, Marzinelli EM, Mason OU, Mayer GD, McDevitt-Irwin JM, McDonald JE, McGuire KL, McMahon KD, McMinds R, Medina M, Mendelson JR, Metcalf JL, Meyer F, Michelangeli F, Miller K, Mills DA, Minich J, Mocali S, Moitinho-Silva L, Moore A, Morgan-Kiss RM, Munroe P, Myrold D, Neufeld JD, Ni Y, Nicol GW, Nielsen S, Nissimov JI, Niu K, Nolan MJ, Noyce K, O’Brien SL, Okamoto N, Orlando L, Castellano YO, Osuolale O, Oswald W, Parnell J, Peralta-Sánchez JM, Petraitis P, Pfister C, Pilon-Smits E, Piombino P, Pointing SB, Pollock FJ, Potter C, Prithiviraj B, Quince C, Rani A, Ranjan R, Rao S, Rees AP, Richardson M, Riebesell U, Robinson C, Rockne KJ, Rodriguezl SM, Rohwer F, Roundstone W, Safran RJ, Sangwan N, Sanz V, Schrenk M, Schrenzel MD, Scott NM, Seger RL, Seguin-Orlando A, Seldin L, Seyler LM, Shakhsheer B, Sheets GM, Shen C, Shi Y, Shin H, Shogan BD, Shutler D, Siegel J, Simmons S, Sjöling S, Smith DP, Soler JJ, Sperling M, Steinberg PD, Stephens B, Stevens MA, Taghavi S, Tai V, Tait K, Tan CL, Tas, N, Taylor DL, Thomas T, Timling I, Turner BL, Urich T, Ursell LK, van der Lelie D, Van Treuren W, van Zwieten L, Vargas-Robles D, Thurber RV, Vitaglione P, Walker DA, Walters WA, Wang S, Wang T, Weaver T, Webster NS, Wehrle B, Weisenhorn P, Weiss S, Werner JJ, West K, Whitehead A, Whitehead SR, Whittingham LA, Willerslev E, Williams AE, Wood SA, Woodhams DC, Yang Y, Zaneveld J, Zarraonaindia I, Zhang Q, Zhao H. 2017. A 
1162 communal catalogue reveals Earth's multiscale microbial diversity. Nature 551:457-463. DOI:

1163

1164

1165

1166

1167

1168

1169

1170

1171

1172

1173

1174

1175

1176

1177

1178

1179

1180

1181

1182

1183

1184

1185

1186

1187

1188

1189

1190

1191

1192

1193

1194

1195

1196

1197

1198

1199

1200

1201

1202

1203

1204

1205

1206

1207

1208

1209

1210

1211

1212 10.1038/nature24621.

Tourlousse DM, Yoshiike S, Ohashi A, Matsukura S, Noda N, Sekiguchi Y, Schoenebeck F, Murphy JA, Zhou S, Uenoyama Y, Miclo Y, Chemistry A, Building TG. 2017. Synthetic spike-in standards for high-throughput 16S rRNA gene amplicon sequencing. Nucleic Acids Res. 45:e23. DOI: 10.1093/nar/gkw984.

Turroni F, Foroni E, Serafini F, Viappiani A, Montanini B, Bottacini F, Ferrarini A, Bacchini PL, Rota C, Delledonne M, Ottonello S, van Sinderen D, Ventura M. 2011. Ability of Bifidobacterium breve to grow on different types of milk: Exploring the metabolism of milk through genome analysis. Applied and Environmental Microbiology 77:7408-7417. DOI: 10.1128/AEM.05336-11.

Turroni F, Peano C, Pass DA, Foroni E, Severgnini M, Claesson MJ, Kerr C, Hourihane J, Murray D, Fuligni F, Gueimonde M, Margolles A, de Bellis G, O’Toole PW, van Sinderen D, Marchesi JR, Ventura M. 2012. Diversity of bifidobacteria within the infant gut microbiota. PLoS ONE 7:20-24. DOI: 10.1371/journal.pone.0036957.

Vandeputte D, Kathagen G, D'hoe K, Vieira-Silva S, Valles-Colomer M, Sabino J, Wang J, Tito RY, De Commer L, Darzi Y, Vermeire S, Falony G, Raes J, D’hoe K, Vieira-Silva S, Valles-Colomer M, Sabino J, Wang J, Tito RY, De Commer L, Darzi Y, Vermeire S, Falony G, Raes J. 2017. Quantitative microbiome profiling links gut community variation to microbial load. Nature 551:507-511. DOI: 10.1038/nature24460.

Vieira-Silva S, Rocha EPC. 2010. The systemic imprint of growth and its uses in ecological (meta)genomics. PLoS Genetics 6. DOI: 10.1371/journal.pgen.1000808.

Virtanen P, Gommers R, Oliphant TE, Haberland M, Reddy T, Cournapeau D, Burovski E, Peterson P, Weckesser W, Bright J, van der Walt SJ, Brett M, Wilson J, Millman KJ, Mayorov N, Nelson ARJ, Jones E, Kern R, Larson E, Carey C, Polat İ, Feng Y, Moore EW, VanderPlas J, Laxalde D, Perktold J, Cimrman R, Henriksen I, Quintero EA, Harris CR, Archibald AM, Ribeiro AH, Pedregosa F, van Mulbregt P, Contributors S 1. 0. 2019. SciPy 1.0--Fundamental algorithms for scientific computing in python. arXiv:1-22.

Wallden M, Fange D, Lundius EG, Baltekin Ö, Elf J. 2016. The synchronization of replication and division cycles in individual E. coli cells. Cell 166:729-739. DOI: 10.1016/j.cell.2016.06.052.

Watanabe S. 2010. Asymptotic equivalence of Bayes cross validation and widely applicable information criterion in singular learning theory. Journal of Machine Learning Research 11:3571-3594.

Watanabe S, Ohbayashi R, Shiwa Y, Noda A, Kanesaki Y, Chibazakura T, Yoshikawa H. 2012. Light-dependent and asynchronous replication of cyanobacterial multi-copy chromosomes. Molecular Microbiology 83:856865. DOI: 10.1111/j.1365-2958.2012.07971.x.

Wendel BM, Courcelle CT, Courcelle J. 2014. Completion of DNA replication in Escherichia coli. Proceedings of the National Academy of Sciences 111:16454-16459. DOI: 10.1073/pnas.1415025111.

Wood DE, Lu J, Langmead B. 2019. Improved metagenomic analysis with Kraken 2. Genome Biology 20. DOI: 10.1186/s13059-019-1891-0.

Wu Z, Liu J, Yang H, Liu H, Xiang H. 2014. Multiple replication origins with diverse control mechanisms in Haloarcula hispanica. Nucleic Acids Research 42:2282-2294. DOI: 10.1093/nar/gkt1214.

Xu J, Yanagisawa Y, Tsankov AM, Hart C, Aoki K, Kommajosyula N, Steinmann KE, Bochicchio J, Russ C, Regev A, Rando OJ, Nusbaum C, Niki H, Milos P, Weng Z, Rhind N. 2012. Genome-wide identification and characterization of replication origins by deep sequencing. Genome Biology 13:R27. DOI: 10.1186/gb-201213-4-r27.

Yang H, Wu Z, Liu J, Liu X, Wang L, Cai S, Xiang H. 2015. Activation of a dormant replication origin is essential for Haloferax mediterranei lacking the primary origins. Nature Communications 6:8321. DOI: $10.1038 /$ ncomms9321.

Yoshikawa H, Wake R. 1993. Initiation and Termination of Chromosome Replication. In: Sonenshein AL, Hoch JA, Losick R eds. Bacillus subtilis and Other Gram-Positive Bacteria. 507-528. DOI: 10.1128/9781555818388.ch36.

Zhou Y, Liang Y, Lynch KH, Dennis JJ, Wishart DS. 2011. PHAST: A fast phage search tool. Nucleic Acids Research 39:347-352. DOI: 10.1093/nar/gkr485.

Zhu A, Sunagawa S, Mende DR, Bork P. 2015. Inter-individual differences in the gene content of human gut bacterial species. Genome Biology 16:82. DOI: 10.1186/s13059-015-0646-9.

1213

Peer) reviewing PDF | (2019:10:42075:3:0:NEW 6 Feb 2020) 


\section{Figure 1}

Effects of growth, sequence feature, and outliers on coverage depth shape

We characterized the coverage depth of chromosomal DNA using statistical models. The shape of the probability distributions (solid lines) and artificial coverage depth (blue lines) obtained using the (a) von Mises, (b) cardioid, (c) wrapped Cauchy, (d) Jones-Pewsey, and (e) linear cardioid distribution model with multinomial distribution. Zero (0) was used as a location parameter, while 0.34657 (von Mises and Jones-Pewsey), 0.16666 (cardioid), 0.17157 (wrapped Cauchy), and 0.1061 (linear cardioid) were used as concentration parameters to align the PPTR with 2.0. The nucleotide number was set to $1 \mathrm{Mnt}$, and the average coverage depth was set to $20 \mathrm{x}$ in the multinomial distribution. For the Jones-Pewsey distribution, 0.5 was used as the shape parameter. Sorted shapes of the distributions and pseudo-coverage depths from the (f) von Mises, (g) cardioid, (h) wrapped Cauchy, (i) JonesPewsey, and (j) linear cardioid distribution model with multinomial distribution. (b) Coverage depth and sequence features that can cause strong noise in the coverage depth of $L$. gasseri (ERR969426). (k) Overall coverage depth, (I) suspected feature-free region, $(\mathrm{m})$ sorted overall coverage depth, and (n) sorted feature-free region. 

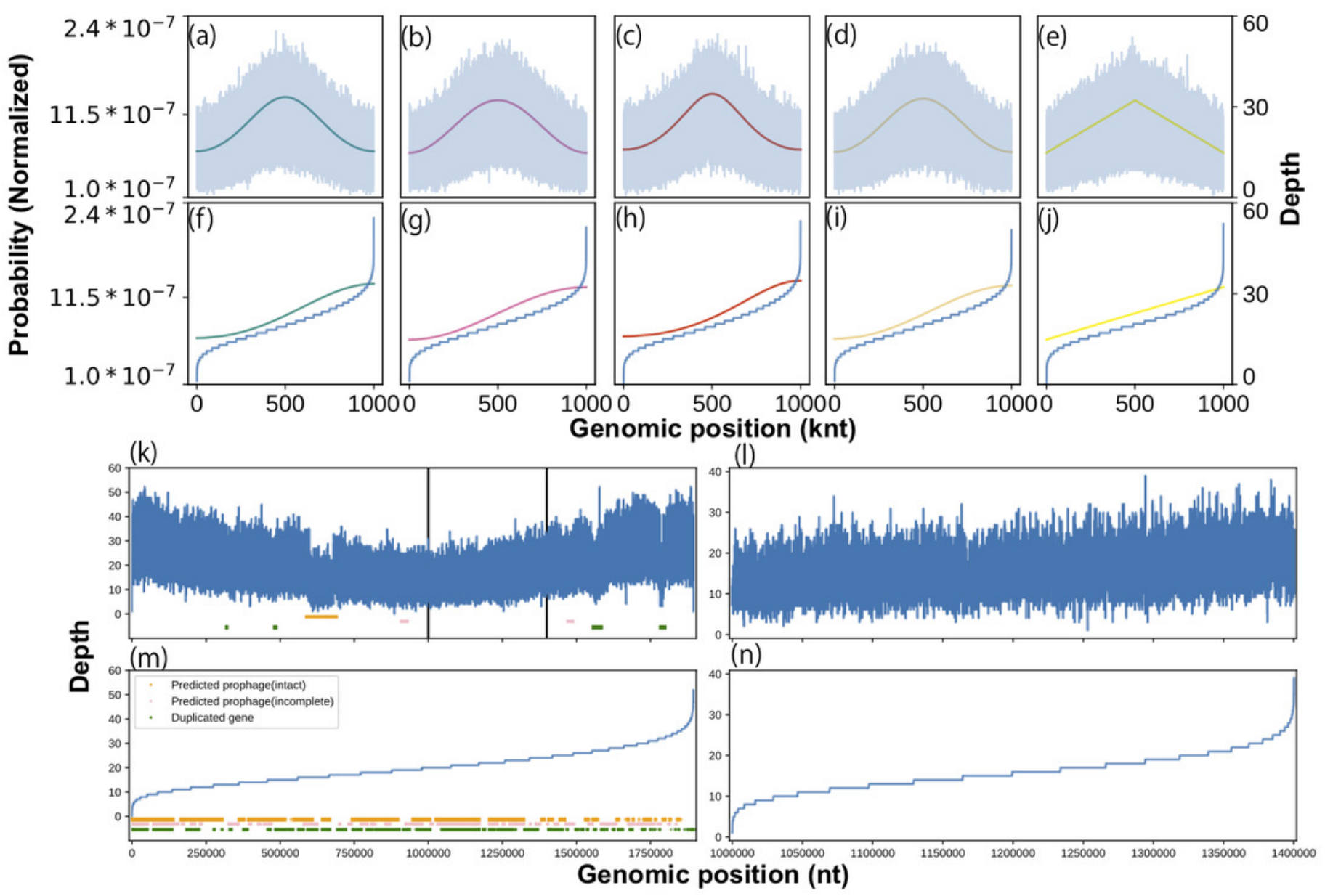


\section{Figure 2}

Probabilistic model for generating coverage depth

Summary of procedures and distributions with coverage. (a) Overall flowchart of our method.

The green parallelogram represents the data, and the pink rectangle represents the procedure. (b) Coverage depth on the circumference. Focusing on the genome structure of prokaryotes, we developed a model that conducted circular regression. The green and pink plots represent the peak and the trough estimated from the model, respectively. (c) Correlation with experimental growth rate by time series aerobic cultured E. coli. PPTR is correlated with the experimental growth rate with a Pearson correlation of 0.964 . The dataset was obtained previously (Korem et al., 2015).

(a)

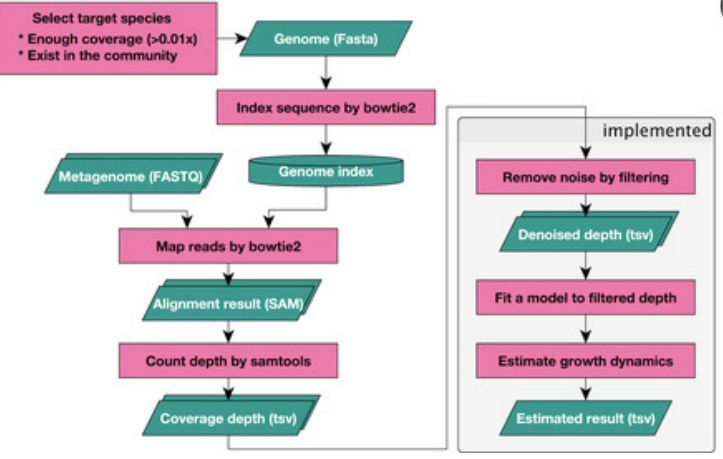

(b)

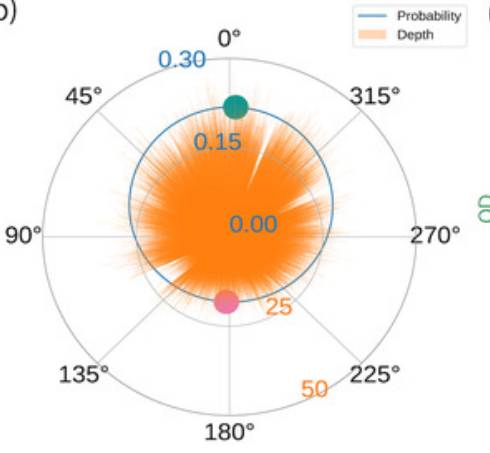

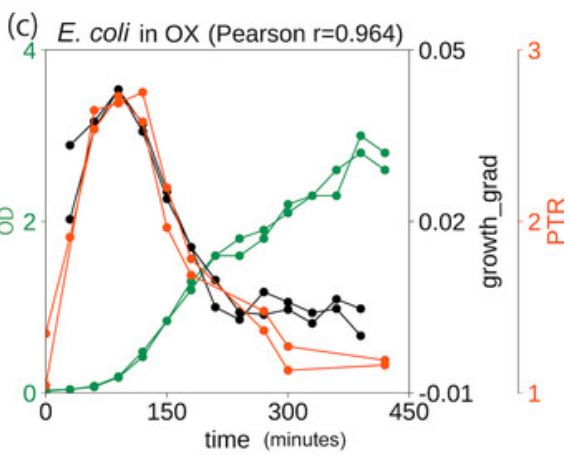


Figure 3

Error rates of growth estimates from various coverage depths with respect to the full coverage depth

The error rates for each average coverage depth were calculated with respect to the full coverage depth. (a) Only the E. coli, E. faecalis, and L. gasseri WGSs with greater than 5.0x coverage, or (b) species with more than $20 \times$ coverage in human fecal WGS datasets were used (Franzosa et al., 2018). The horizontal bar represents the $15 \%$ threshold of the error rate threshold. The black crosses on the bar represent the unavailability of the methods with respect to the coverage depth. The proposed models and statistics are shown in the black rectangle within the legend.

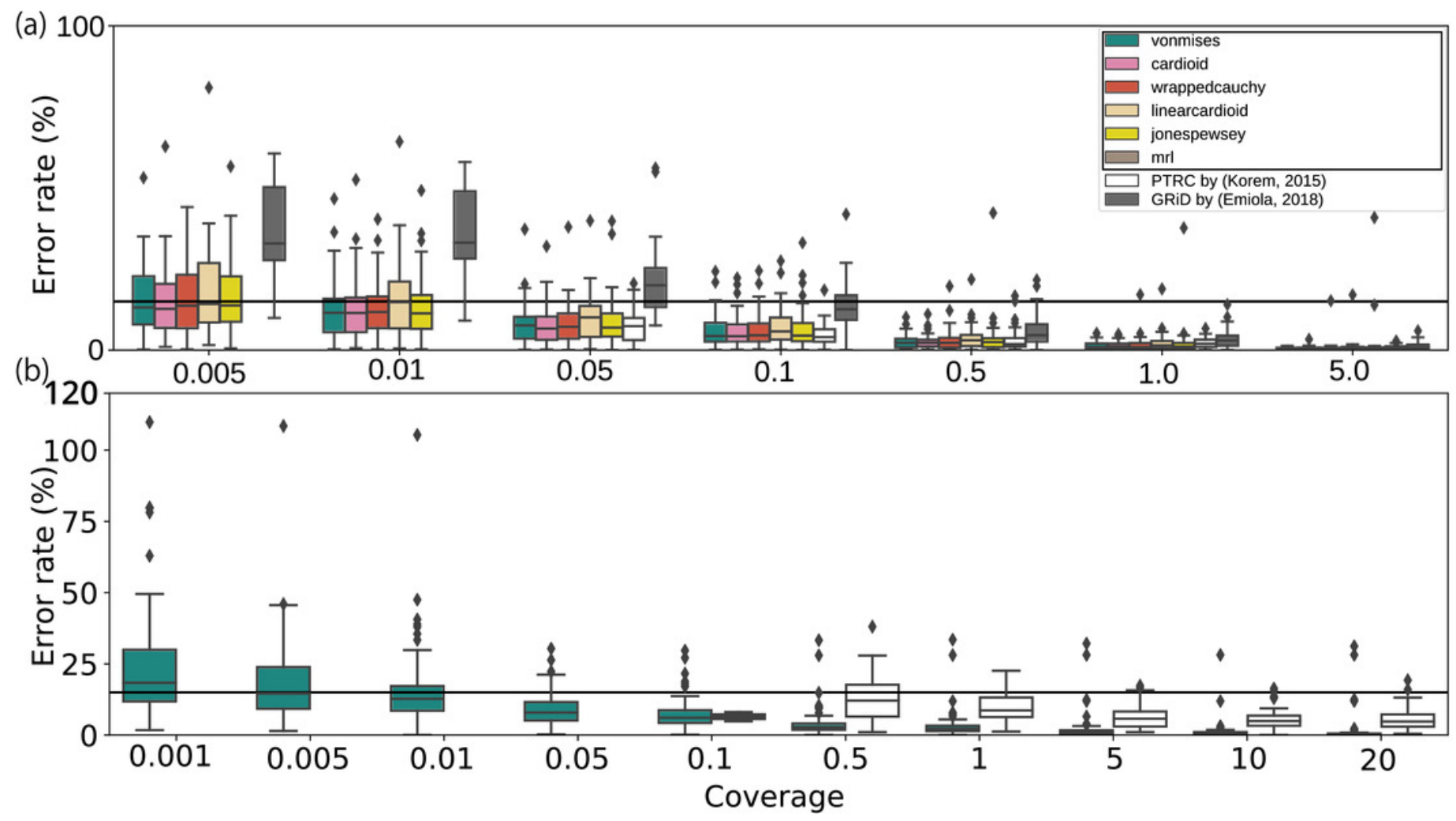


Figure 4

Replication peakedness

The InvSE von Mises distribution-based model exhibits a tapered shape of the coverage depth trend. The Jones-Pewsey distribution-based model also shows a concentrated shape.

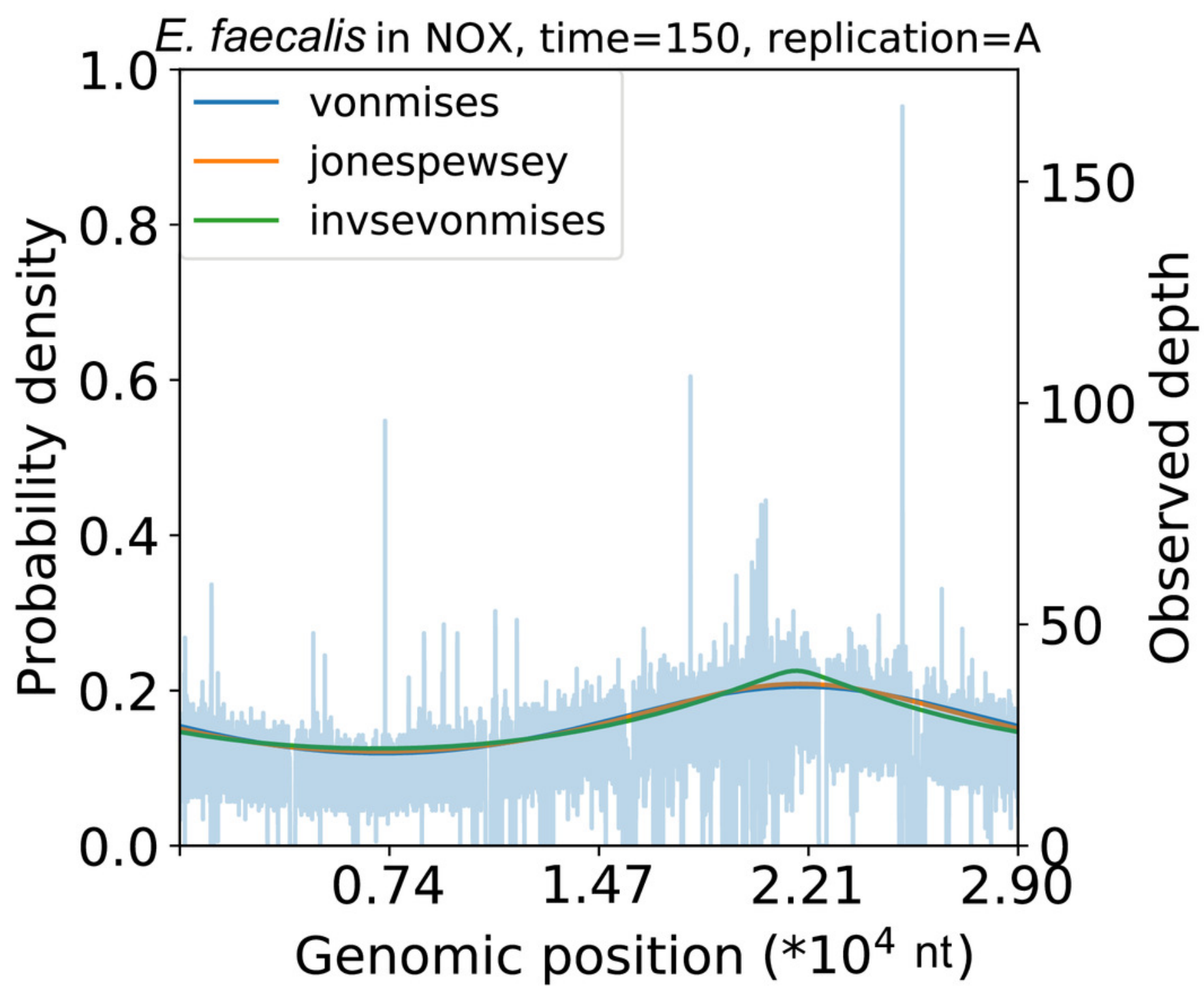




\section{Figure 5}

Extension to multiple replication origins

The plots in the upper row represent the coverage depth and probability distributions estimated using a mixture of the von Mises distribution models for Haloferax volcanii in the (a) exponential growth phase, and (b) stationary phase. The blue lines represent the coverage depth processed using a median filter with $100 \mathrm{nt}$ for both the stride and window length. The black arrows indicate the position of $c d c 6$ in the genome. The lines inside the lower circular plot express the magnitude of the weighted pPTR for $\mathrm{H}$. volcanii in the (c) exponential growth phase and (d) stationary phase. The circles represent positions of replication origins, and the crosses represent the positions of the centers of gravity of the replication origins. The lines inside the circles represent the relative magnitudes of the weighted PPTR. 
(a)

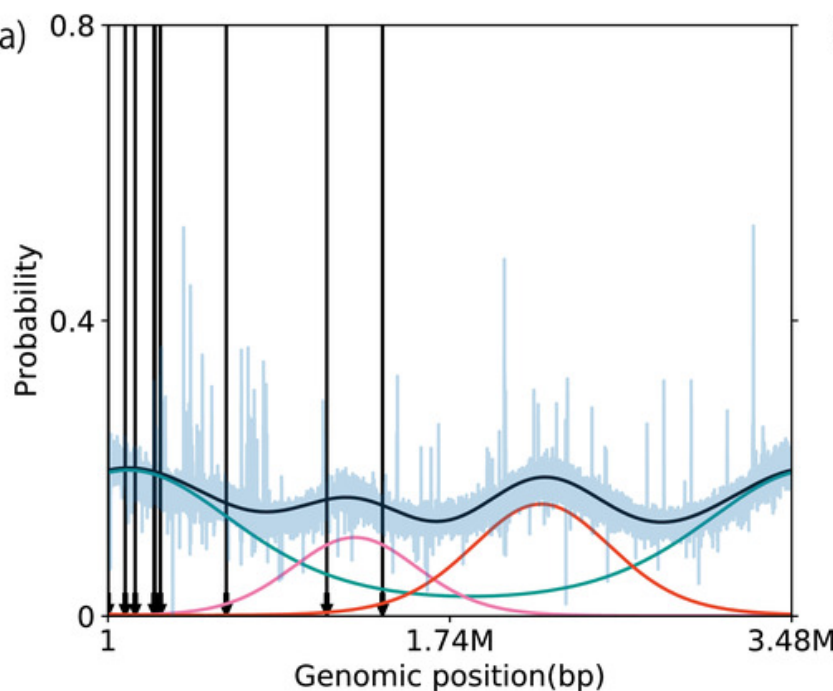

(b)

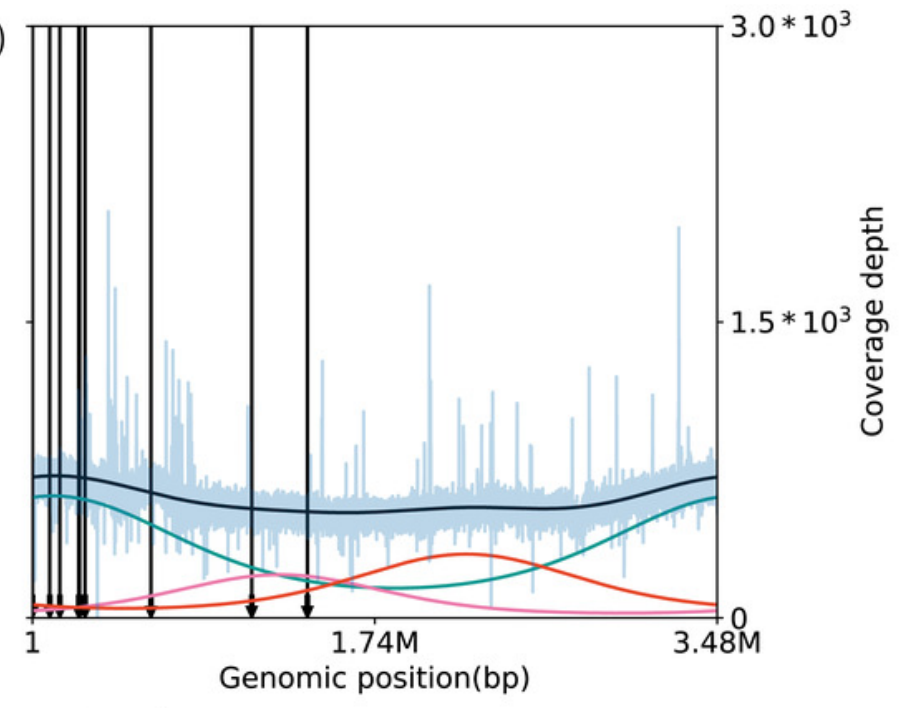

(c)

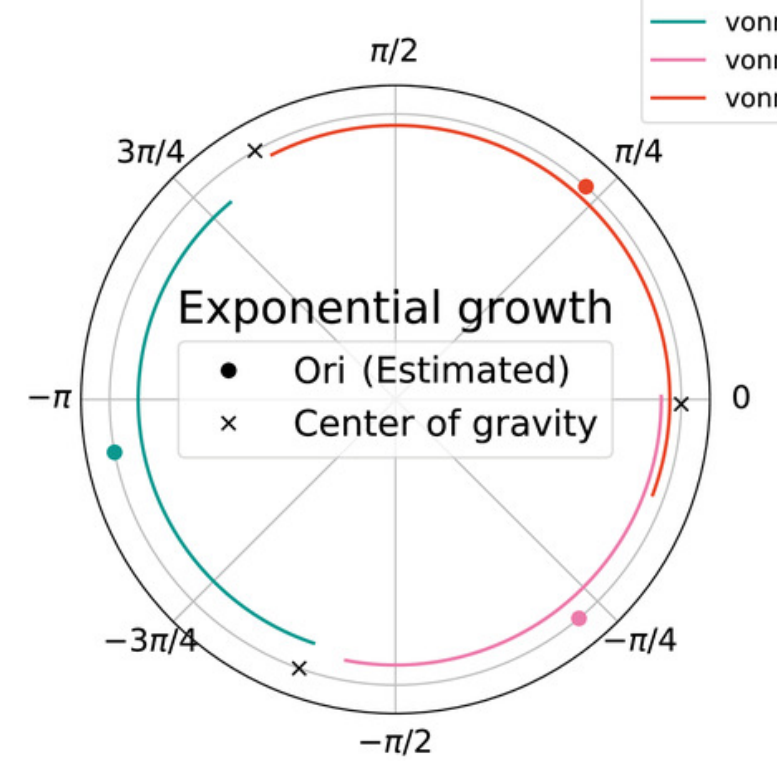

(d)

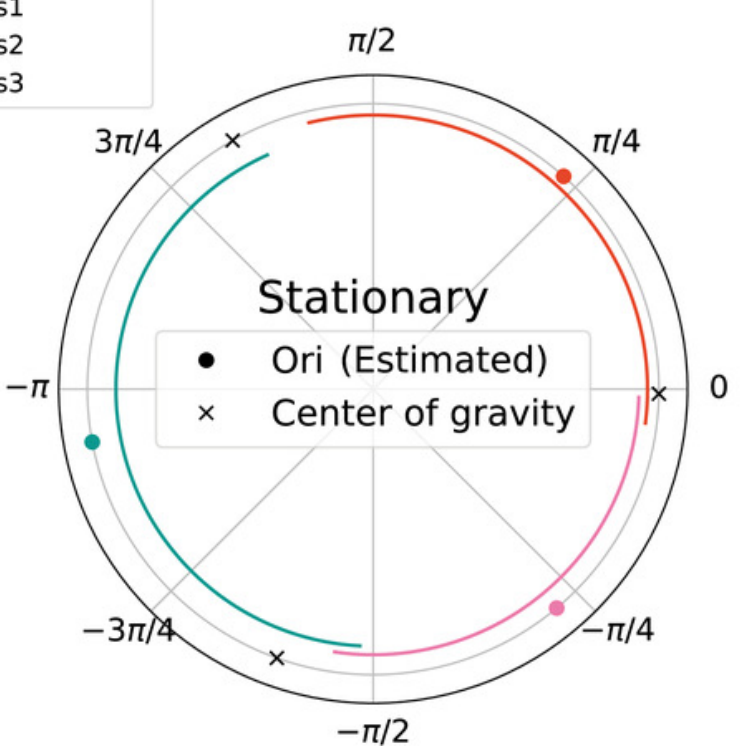




\section{Table $\mathbf{1}$ (on next page)}

Circular distributions for modeling coverage depth dynamics.

mu: location parameter, kappa or rho: concentration parameter, psi: shape parameter, $I_{0}$ : modified Bessel function of the first kind of order $0, P_{-}\{1 / p s i\}$ : the associated Legendre function of the first kind of degree 1/psi, theta: an angle converted from the observed position, $T$ : total number of observations. 
1 Table1: Circular distributions for modeling coverage depth dynamics. $\mu$ : location parameter, $\kappa$ or $2 \rho$ : concentration parameter, $\psi$ : shape parameter, $I_{0}$ : modified Bessel function of the first kind of 3 order $0, P_{\frac{1}{\psi}}$ : the associated Legendre function of the first kind of degree $\frac{1}{\psi}, \theta$ : an angle converted

4 from the observed position, $T$ : total number of observations.

\begin{tabular}{|c|c|c|c|c|}
\hline Name & (Probability density) function & pPTR & Parameters & Ref. \\
\hline \multirow[t]{2}{*}{ von Mises (vM) } & $\exp (\kappa \cos (\theta-\mu))$ & $\exp (2 \kappa)$ & $\mu, \kappa$ & \\
\hline & $2 \pi I_{0}(\kappa)$ & & & \\
\hline \multirow[t]{2}{*}{ Cardioid (c) } & $1+2 \rho_{\mathrm{c}} \cos (\theta-\mu)$ & $1+2 \rho_{\mathrm{c}}$ & $\mu, \rho_{\mathrm{c}}$ & \\
\hline & $2 \pi$ & $\overline{1-2 \rho_{\mathrm{c}}}$ & & \\
\hline \multirow{2}{*}{$\begin{array}{l}\text { Wrapped Cauchy } \\
(\mathrm{wC})\end{array}$} & $1-\rho_{\mathrm{wC}}^{2}$ & $\left(1+\rho_{\mathrm{wC}}\right)^{2}$ & $\mu, \rho_{\mathrm{wC}}$ & \\
\hline & $\overline{2 \pi\left(1+\rho_{\mathrm{wC}}{ }^{2}-2 \rho_{\mathrm{wC}} \cos (\theta-\mu)\right)}$ & $\overline{\left(1-\rho_{\mathrm{wC}}\right)^{2}}$ & & \\
\hline \multirow[t]{3}{*}{ Jones-Pewsey (JP) } & 1 & $\exp (2 \kappa)$ & $\mu, \kappa, \psi$ & (Jones \& \\
\hline & $(\cosh (\kappa \psi)+\sinh (\kappa \psi) \cos (\theta))^{\bar{\psi}}$ & & & Pewsey, 2005) \\
\hline & $2 \pi P_{\frac{1}{\psi}}(\cosh (\kappa \psi))$ & & & \\
\hline \multirow{3}{*}{$\begin{array}{l}\text { Linear cardioid } \\
\text { (lc) }\end{array}$} & $1+20\left(|| \theta-u|-\pi|-\frac{\pi}{2}\right)$ & $1+\pi \rho_{\mathrm{lc}}$ & $\mu, \rho_{\mathrm{lc}}$ & \\
\hline & $1+2 \rho_{\operatorname{lc}}(|| \sigma-\mu|-\pi|-\overline{2})$ & $\overline{1-\pi \rho_{\mathrm{lc}}}$ & & \\
\hline & $2 \pi$ & & & \\
\hline \multirow[t]{2}{*}{$\begin{array}{l}\text { Exponential linear } \\
\text { cardioid (elc) }\end{array}$} & $\rho_{\text {elc }} \exp \left(2 \rho_{\text {elc }}|| \theta-\mu|-\pi|-\frac{\pi}{2}\right)$ & $\exp \left(2 \pi \rho_{\text {elc }}\right)$ & $\mu, \rho_{\text {elc }}$ & \\
\hline & $\exp \left(\pi \rho_{\text {elc }}\right)-\exp \left(-\pi \rho_{\text {elc }}\right)$ & & & \\
\hline \multirow[t]{2}{*}{$\begin{array}{l}\text { Mean resultant } \\
\text { length }(\mathrm{mrl})\end{array}$} & $\sqrt{\left(\sum_{t=1}^{T} \cos \theta_{t}\right)^{2}+\left(\sum_{t=1}^{T} \sin \theta_{t}\right)^{2}}$ & & & \\
\hline & $T$ & & & \\
\hline
\end{tabular}

